\title{
Vesicular GABA Transporter Is Necessary for Transplant- Induced Critical Period Plasticity in Mouse Visual Cortex
}

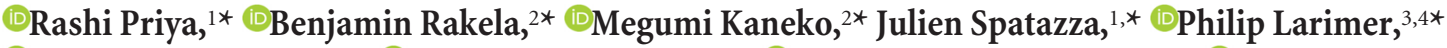 \\ ${ }^{\circledR}$ Mahmood S. Hoseini, ${ }^{2 *}{ }^{\circledR}$ Andrea R. Hasenstaub, ${ }^{3}{ }^{\circledR}$ Arturo Alvarez-Buylla, ${ }^{1}$ and ${ }^{\circledR}$ Michael P. Stryker ${ }^{2}$ \\ ${ }^{1}$ Department of Neurological Surgery and the Eli and Edythe Broad Center of Regeneration Medicine and Stem Cell Research, ${ }^{2}$ Center for Integrative \\ Neuroscience and Department of Physiology, ${ }^{3}$ Center for Integrative Neuroscience and Department of Otolaryngology-Head and Neck Surgery, \\ and ${ }^{4}$ Department of Neurology, University of California, San Francisco, California 94143
}

The maturation of GABAergic inhibitory circuits is necessary for the onset of the critical period for ocular dominance plasticity (ODP) in the postnatal visual cortex (Hensch, 2005; Espinosa and Stryker, 2012). When it is deficient, the critical period does not start. When inhibitory maturation or signaling is precocious, it induces a precocious critical period. Heterochronic transplantation of GABAergic interneuron precursors derived from the medial ganglionic eminence (MGE) can induce a second period of functional plasticity in the visual cortex (Southwell et al., 2010). Although the timing of MGE transplantation-induced plasticity is dictated by the maturation of the transplanted cells, its mechanisms remain largely unknown. Here, we sought to test the effect of blocking vesicular GABA loading and subsequent release by transplanted interneurons on the ability to migrate, integrate, and induce plasticity in the host circuitry. We show that MGE cells taken from male and female donors that lack vesicular GABA transporter ( Vgat) expression disperse and differentiate into somatostatin- and parvalbumin-expressing interneurons upon heterochronic transplantation in the postnatal mouse cortex. Although transplanted Vgat mutant interneurons come to express mature interneuron markers and display electrophysiological properties similar to those of control cells, their morphology is significantly more complex. Significantly, Vgat mutant MGE transplants fail to induce ODP, demonstrating the pivotal role of vesicular GABAergic transmission for MGE transplantation-induced plasticity in the postnatal mouse visual cortex.

Key words: critical period; GABA; medial ganglionic eminence; transplantation; VGAT; visual cortex

Significance Statement

Embryonic inhibitory neurons thrive when transplanted into postnatal brains, migrating and differentiating in the host as they would have done if left in the donor. Once integrated into the host, these new neurons can have profound effects. For example, in the visual cortex, such neurons induce a second critical period of activity-dependent plasticity when they reach the appropriate stage of development. The cellular mechanism by which these transplanted GABAergic interneurons induce plasticity is unknown. Here, we show that transplanted interneurons that are unable to fill synaptic vesicles with GABA migrate and integrate into the host circuit, but they do not induce a second period of plasticity. These data suggest a role for the vesicular GABA transporter in transplantation-mediated plasticity.

\section{Introduction}

GABAergic inhibitory neurons modulate neural circuit activity throughout the CNS (Wehr and Zador, 2003; Yizhar et al., 2011).

Received May 17, 2018; revised Jan. 7, 2019; accepted Jan. 8, 2019.

Author contributions: R.P. wrote the first draft of the paper; B.R., M.K., P.L., M.S.H., A.R.H., A.A.-B., and M.P.S. edited the paper; R.P., M.K., J.S., A.R.H., A.A.-B., and M.P.S. designed research; R.P., B.R., M.K., J.S., P.L., and M.S.H. performed research; R.P., B.R., M.K., M.S.H., A.R.H., A.A.-B., and M.P.S. analyzed data; B.R., M.S.H., A.A.-B., and M.P.S. wrote the paper.

This work was supported by the National Institutes of Health (Grants R01EY025174 and R01DC014101). M.P.S. is a recipient of the Research to Prevent Blindness Stein Innovator Award. A.A.-B. is a Heather and Melanie Muss Endowed Chair and has been generously supported by the John G. Bowes Research Fund. A.R.H. has been supported by the Coleman Memorial Fund, Hearing Research, Inc., the Klingenstein Foundation, and the UCSF Program in
In mice, they comprise $\sim 20 \%$ of cortical neurons and originate largely from the embryonic caudal ganglionic eminence (CGE) and the medial ganglionic eminence (MGE) (Anderson et al., 1997; Pleasure et al., 2000; Wichterle et al., 2001; Nery et al., 2002;

Breakthrough Biomedical Research. We thank members of the Stryker, Hasenstaub, and Alvarez-Buylla laboratories for helpful discussions.

A.A.-B. is cofounder and serves on the scientific advisory board and J.S. is an employee of Neurona Therapeutics; both own shares in the company. The remaining authors declare no competing financial interests.

${ }^{*}$ R.P., B.R., M.K., J.S., P.L., and M.S.H. contributed equally to this work.

J. Spatazza's present address: Neurona Therapeutics, South San Francisco, CA 94080.

Correspondence should be addressed to Michael P. Stryker at stryker@phy.ucsf.edu.

https://doi.org/10.1523/JNEUROSCI.1253-18.2019

Copyright $\odot 2019$ the authors 
Wonders and Anderson, 2006). Cells derived from the MGE and CGE migrate to specific laminae in the developing neocortex and differentiate into distinct types of GABAergic interneurons (Anderson et al., 1999, 2001; Parnavelas, 2000; Wichterle et al., 2001; Nery et al., 2002; Butt et al., 2005). MGE- and CGE-derived interneurons retain the ability to disperse and functionally integrate when transplanted into both the juvenile and adult brain, receiving excitatory and inhibitory inputs and forming inhibitory synapses onto host neurons (Wichterle et al., 1999; AlvarezDolado et al., 2006; Southwell et al., 2010; Larimer et al., 2016, 2017). Modifying synaptic inhibition by MGE-derived interneuron transplantation provides a unique opportunity for the treatment of disorders that are linked to interneuron dysfunction or circuit hyperexcitability (Southwell et al., 2014; Tyson and Anderson, 2014; Spatazza et al., 2017).

The mouse visual cortex exhibits dramatic ocular dominance plasticity (ODP) during a brief critical period beginning at approximately postnatal day 26 (P26) to P28, when cortical inhibitory neurons are 33-35 d old. During this period, occluding the vision of one eye rapidly reduces its ability to drive cortical cells and causes a permanent loss of its visual acuity (Hensch, 2005). This plasticity is mediated by the maturation of cortical GABAergic interneurons (Hensch, 2005). Mice deficient in GAD65, one of the two enzymes that synthesize GABA, release only half the normal amount of GABA and fail to undergo experiencedependent plasticity upon brief monocular deprivation (Hensch et al., 1998). ODP can be rescued in these animals by cortical administration of diazepam, which doubles the efficacy of GABAergic signaling. The effect of pharmacological intervention is limited by the timing of the critical period; diazepam application in WT animals after the normal critical period does not initiate a new period of plasticity (Fagiolini and Hensch, 2000). However, ODP in WT mice can be triggered 1 week earlier than the normal time by promoting precocious development of GABAergic interneurons (Hanover et al., 1999; Di Cristo et al., 2007; Sugiyama et al., 2008) or by pharmacologically enhancing GABAergic transmission in the visual cortex (Fagiolini and Hensch, 2000; Fagiolini et al., 2004), in which case there is no additional critical period at the normal time.

Given the importance of GABAergic signaling in cortical development and in mediating critical period plasticity, GABAergic interneurons are ideal candidates for reintroducing periods of plasticity. Interestingly, 33-35 d after transplantation (DAT) into juvenile or adult visual cortex, MGE-derived (but not CGEderived) GABAergic interneurons have the ability to reinstate a new period of ODP that resembles the normal critical period (Southwell et al., 2010; Tang et al., 2014; Davis et al., 2015; Larimer et al., 2016; Isstas et al., 2017). This timing suggests that transplantation-induced plasticity is linked to the maturation of the transplanted inhibitory interneurons. However, the mechanisms by which the grafted MGE-derived interneurons induce plasticity have not been elucidated.

Here, we tested the role of vesicular GABA release in the maturation of transplanted interneurons, their integration into the recipient visual cortex, and their capacity to induce a second period of plasticity. We used the Cre/loxP system to delete the vesicular GABA transporter (VGAT, encoded by Vgat, also known as Slc32a1) in MGE-derived interneurons. Transplanted MGE-derived interneurons deficient in VGAT dispersed, differentiated, and matured appropriately in the host brain. VGAT deficiency did not prevent the expression of perineuronal nets or Orthodenticle homeobox 2 protein (Otx2) in transplanted neurons, but did increase their axonal length and branching. Despite their successful engraftment, MGE-derived transplanted interneurons with loss of VGAT function were incompetent to induce plasticity following monocular deprivation.

\section{Materials and Methods}

Animals. R26-Ai14, Vgat ${ }^{\mathrm{fl} / \mathrm{fl}}$, Gad2-cre, Nkx2.1-cre, and WT C57BL/6J breeders were purchased from The Jackson Laboratory (stock numbers 071914, 012897, 010802, 08661, and 00664, respectively). Both males and females were used for all experiments. All animals were treated in accordance with the regulations and guidelines of the Institutional Animal Care and Use Committees at the University of California-San Francisco.

Cell dissection and transplantation. MGE were dissected from embryonic day 13.5 (E13.5) to E14.5 donor embryos, mechanically dissociated, concentrated via centrifugation $(800 \times g$ for $3 \mathrm{~min})$, and front-loaded into beveled glass micropipettes $(\approx 50 \mu \mathrm{m}$ diameter) prefilled with mineral oil and mounted on a microinjector. Transplantation was performed into anesthetized (on ice for $3 \mathrm{~min}$ ) mouse pups (P2-P6). Unilateral injections ( $\approx 4 \times 10^{5}$ cells per mouse) were made into the cortex at two different sites in one hemisphere as described previously (Tang et al., 2014).

Immunostaining. Brains were fixed by transcardiac perfusion followed by $1 \mathrm{~h}$ of postfixation on ice with $4 \%$ formaldehyde/PBS solution. Brains were rinsed with PBS and cryoprotected by using 30\% sucrose/PBS solution overnight at $4^{\circ} \mathrm{C}$. Immunohistochemistry was performed on floating $50 \mu \mathrm{m}$ sections using a PBS solution containing $1.5 \%$ normal goat serum and $0.25 \%$ Triton X-100 for all procedures. The washing steps were done with PBS. The sections were incubated overnight at $4^{\circ} \mathrm{C}$ with selected antibodies, followed by incubation at $4^{\circ} \mathrm{C}$ overnight with donkey secondary antibodies (Jackson ImmunoResearch Laboratories). For cell counting and post hoc examination of marker expression, sections were stained using chicken anti-GFP (1:1000, Aves Labs, GFP-1020, RRID:AB_10000240), mouse anti-Reelin (1:500 MBL, D223-3, RRID:AB_843523), rabbit anti-dsRed (1:1000 Abcam, ab62341, RRID:AB_945213), mouse anti-parvalbumin (anti-PV, 1:500, Sigma-Aldrich, P3088, RRID:AB_477329), and rat anti-somatostatin (SST, 1:500, Santa Cruz Biotechnology, sc-7819, RRID:AB_2302603), goat anti-Otx2 (1:500, R\&D Systems, AF1979, RRID:AB_2157172), rabbit anti- VGAT (1:500, Millipore, ab5062p, RRID:AB_2301998), biotinylated Wisteria floribunda agglutinin (WFA, 1:7000, Vector Laboratories, B-1355, RRID:AB_2336874). For cell culture immunostaining, the plate was fixed with $4 \%$ paraformaldehyde and then washed with PBS before blocking with $0.1 \%$ BSA in $1 \times$ PBS. Culture cells were incubated in primary antibody (Rb-VGAT antibody, 1:500, Millipore, ab5062p, RRID:AB_2301998) overnight at $4^{\circ} \mathrm{C}$. The following day, the primary was replaced by the secondary antibody and was incubated for 3-4 h at room temperature. The slides were then washed and mounted with mounting medium.

Cell counting. Cell density was defined as the number of fluorescent cells per square millimeter in a given coronal section within the binocular visual cortex, which was identified functionally during intrinsic signal imaging sessions. Three alternate coronal sections were selected along the rostral-caudal axis. Value for each section was calculated and these numbers were then averaged, accounting for cell density for each animal.

Neuronal morphology analysis. Neuronal morphology was obtained by labeling the transplanted MGE-derived cortical interneurons (CINs) using tdTomato. Successful labeling of the MGE subtypes in all of our experiments was confirmed through post hoc examination of immunohistochemical marker expression. Neuronal morphology was reconstructed using Neurolucida (MicroBrightField) from a single 50$\mu \mathrm{m}$-thick section imaged in a confocal stack taken at $1 \mu \mathrm{m}$ intervals. Morphometric analysis was done using Neurolucida Explorer for both axonal and dendritic length and branching.

Slice electrophysiology. Host animals were overdosed with pentobarbital and decapitated at 33-45 DAT. The brain was removed into ice-cold dissection buffer containing the following (in $\mathrm{mm}$ ): 234 sucrose, $2.5 \mathrm{KCl}$, $10 \mathrm{MgSO}_{4}, 1.25 \mathrm{NaH}_{2} \mathrm{PO}_{4}, 24 \mathrm{NaHCO}_{3}, 11$ dextrose, and $0.5 \mathrm{CaCl}_{2}$ bubbled with $95 \% \mathrm{O}_{2} / 5 \% \mathrm{CO}_{2}$ to a $\mathrm{pH}$ of 7.4 . Coronal slices of visual cortex (300 $\mu \mathrm{m}$ thick) were cut via a vibratome (Precisionary Instruments) and transferred to artificial CSF (ACSF) containing the following 
(in mM): $124 \mathrm{NaCl}, 3 \mathrm{KCl}, 2 \mathrm{MgSO}_{4}, 1.23 \mathrm{NaH}_{2} \mathrm{PO}_{4}, 26 \mathrm{NaHCO}_{3}, 10$ dextrose, and $2 \mathrm{CaCl}_{2}$ bubbled with $95 \% \mathrm{O}_{2} / 5 \% \mathrm{CO}_{2}$, incubated at $33^{\circ} \mathrm{C}$ for $30 \mathrm{~min}$, then stored at room temperature. The following fluorescently identified transplantation-derived MGE-lineage interneurons were viewed using an IR-DIC video microscope (Olympus BX50WI): either Gad2-Cre or Nkx2.1-Cre; Ai14; and one of the following combinations of $V g a t$ alleles: $V g a t^{+/+}$or $V g a t^{\text {flox/+ }}$ or $V g a t^{\text {flox/flox }}$. Whole-cell currentclamp recordings were made with a Multiclamp 700B (Molecular Devices) using an internal solution containing the following (in $\mathrm{mM}$ ): 140 K-gluconate, $2 \mathrm{MgCl}_{2}, 10 \mathrm{HEPES}, 0.2$ EGTA, $4 \mathrm{MgATP}$, 0.3 NaGTP, 10 phosphocreatine, $\mathrm{pH}$ 7.3, $290 \mathrm{mOsm}$. Data were low-pass filtered at 2.6 $\mathrm{kHz}$ and digitized at $10 \mathrm{kHz}$ by a 16 bit analog-to-digital converter (National Instruments). Data acquisition and analysis were done with custom software written in MATLAB (The MathWorks). We used multivariate ANOVA with Benjamini-Hochberg post hoc correction as implemented by MATLAB for determination of statistical significance for all comparisons of physiological properties.

Monocular deprivation. A custom titanium plate for head fixation was attached to the skull with dental acrylic under anesthesia with isoflurane as described previously (Kaneko and Stryker, 2014) on 28-30 DAT, followed 3-4 d later by baseline recording of intrinsic signal responses in the binocular visual cortex of the transplanted hemisphere. Monocular deprivation was started on 33-35 DAT by suturing shut the eyelid on the side contralateral to the transplanted hemisphere under anesthesia with isoflurane as described below. A second recording of intrinsic signal responses in the same hemisphere was performed after $4 \mathrm{~d}$ of monocular deprivation to evaluate plasticity.

Optical imaging of intrinsic signals. Optical imaging of intrinsic signals was performed as described previously (Kaneko and Stryker, 2014). Briefly, images of the response to a visual stimulus in the binocular visual cortex contralateral to the deprived eye were recorded transcranially through a hole in the implanted head plate in a mouse anesthetized with isoflurane (3\% for induction and $0.8 \%$ during recording) supplemented with intramuscular injection of chlorprothixene chloride $(2 \mu \mathrm{g} / \mathrm{g}$ body weight). Intrinsic signal images were obtained with a CCD camera (1M30; Dalsa) with a $135 \times 50 \mathrm{~mm}$ tandem lens (Nikon). Frames were acquired at a rate of 30 frames/s, temporally binned by 4 frames, and stored as $512 \times 512$ pixel images after binning the $1024 \times 1024$ camera pixels by $2 \times 2$ pixels spatially. Visual stimulus was contrast-modulated Gaussian noise with a randomly generated spatiotemporal spectrum having low-pass spatial and temporal cutoffs applied at $0.05 \mathrm{cpd}$ and 4 $\mathrm{Hz}$, respectively, generated in MATLAB (Niell and Stryker, 2008) using Psychophysics Toolbox extensions (Brainard, 1997; Pelli, 1997). The visual stimulus was presented between $-5^{\circ}$ and $25^{\circ}$ azimuth and $-15^{\circ}$ and $440^{\circ}$ on a monitor (Dell, $30 \times 40 \mathrm{~cm}, 60 \mathrm{~Hz}$ refresh rate, $32 \mathrm{~cd} / \mathrm{m}^{2}$ mean luminance) placed $25 \mathrm{~cm}$ from the mouse with its center aligned to the center of the mouse $\left(=0^{\circ}\right.$ azimuth). The phase and amplitude of cortical responses at the stimulus frequency were extracted by Fourier analysis as described previously (Kalatsky and Stryker, 2003). Response amplitude was an average of at least four measurements through each eye. Ocular dominance index (ODI) was calculated as $(C-I) /(C+I)$, where $C$ and $I$ are the response amplitudes through the eyes of contralateral and ipsilateral, respectively, to the hemisphere imaged using a custom MATLAB code as described previously (Cang et al., 2005).

Experimental design and statistical analysis. The experiments reported here were designed to determine the following: (1) whether defective vesicular GABA release would impair the migration and differentiation of embryonic MGE-derived interneurons when transplanted into postnatal cortex, (2) whether the transplanted neurons would be associated with perineuronal nets (PNNs) and would accumulate Otx2, (3) whether their axonal and dendritic growth would be impaired, (4) whether their intrinsic physiological properties would be abnormal, and (5) whether they would stimulate a second critical period of ODP. The statistical tests are specific for each of these questions. For question (1), the significant source of variability is the individual mouse and the overlap of the raw proportions does not suggest any significant difference. All data are illustrated in the scatter plots of Figures 1 and 3. For question (2), the significant source of variability is the individual transplanted neuron in relation to the surrounding host neurons; here, the ANOVA is the ap- propriate test. All data are shown in the Figure 4 scatter plots. For question (3), the significant source of variability is the individual transplanted neuron in relation to transplanted neurons of different genotypes. The differences were tested with the nonparametric Mann-Whitney $U$ test using the Bonferroni correction for multiple comparisons. All data are shown in the Figure 6 scatter plots. For question (4), the significant source of variability is also the individual transplanted neuron in relation to transplanted neurons of different genotypes. All data are shown in the scatter plots of Figure 7 and the sole feature with significant differences was confirmed by ANOVA. For question (5), the significant source of variability is the individual mouse and the differences between genotypes were tested with the nonparametric Mann-Whitney $U$ test using the Bonferroni correction for multiple comparisons. All data are shown in the scatter plots of Figure 8.

\section{Results}

Transplanted MGE-derived cortical interneurons with VGAT loss of function disperse and differentiate in the host brain

To test whether vesicular GABA release from transplanted MGE cells is required for MGE transplantation-induced plasticity, the $V g a t^{\text {flox/flox }}$ mouse line was used for conditional Vgat loss-offunction experiments. VGAT is required for the loading of GABA into synaptic vesicles and is therefore essential for the normal synaptic release of GABA. Vgat flox/flox animals harbor two flox cassettes flanking the second exon of the Vgat gene (Fig. 1A), which encodes for the putative 10-transmembrane domain of the VGAT protein (Ebihara et al., 2003), the deletion of which leads to disruption of synaptic GABA release (Tong et al., 2008). Donor embryos lacking either one or both copies of Vgat in MGE progenitors were generated by crossing $V g a t^{\text {flox/flox }}$ mice with Nkx2.1 ${ }^{\mathrm{Cre}}:: V_{\text {gat }} t^{\text {flox/+ }}$ mice (Xu et al., 2008). MGE cells obtained from this breeding strategy will be referred to as $\mathrm{Vgat}^{+/-}$and $\mathrm{Vgat}^{-1-}$. The donor animals also carried the Cre-dependent tdTomato reporter allele R26-Ai14 to allow the visualization of the cells in which Cre-mediated recombination occurred. It is well established that synaptic GABA release is absent in cells of the floxed Vgat mouse line that express cre recombinase (Wojcik et al., 2006; Tong et al., 2008; Saito et al., 2010; Vong et al., 2011; Wu et al., 2012; Pei et al., 2015; Hirano et al., 2016; Lin et al., 2018; Weaver et al., 2018). Loss of VGAT from presynaptic regions in MGE-derived interneurons was further assessed in vitro using immunohistochemistry of long-term cultures of $\mathrm{Vgat}^{+/-}$and $\mathrm{Vgat}^{-1-}$ MGE cells. After $20 \mathrm{~d}$ in culture, dense VGAT punctae were observed in the neurites of $\mathrm{Vgat}^{+/-}$cells, whereas puncta were not stained in $\mathrm{Vgat}^{-1-}$ cells, confirming loss of VGAT in synaptic terminals (Fig. 2).

We next investigated whether Vgat $^{-1-}$ MGE cells disperse and differentiate into interneurons of their lineage when transplanted into the cortex of P2-P6 mice. Nkx2.1-cre;Ai14 WT for the Vgat locus $\left(\right.$ Vgat $\left.^{+/+}\right), \mathrm{Vgat}^{+/-}$, and $\mathrm{Vgat}^{-{ }^{-}}$MGE cells were collected from E13.5 donor embryos and transplanted just medial and lateral to the visual cortex of P2-P6 WT hosts (Fig. 1B) (Tang et al., 2014). At P46, host brain sections were immunostained for tdTomato and MGE-lineage interneuron markers PV and SST to characterize the differentiation, spatial distribution, and molecular identity of transplanted interneurons (Fig. $1 C, D, E)$. Transplanted $V_{g a t}{ }^{+/+}$, Vgat $^{+/-}$, and Vgat $^{-1-}$ MGE cells displayed similar proportions of $\mathrm{SST}^{+}$and $\mathrm{PV}^{+}$cells (Fig. 1E) (SST: Vgat $^{+/+}, 42.33 \pm 3.48 \%$ transplanted cells, $n=3$ animals; Vgat $^{+1-}, 41 \pm 2.45 \%$ transplanted cells, $n=5$ animals; Vgat $^{-/-}, 44.28 \pm 2.21 \%$ transplanted cells, $n=9$ animals, oneway ANOVA, $p=0.6386$. PV: Vgat $^{+/+}, 39.67 \pm 7.13 \%$ transplanted cells, $n=3$ animals; Vgat $^{+/-}, 35.29 \pm 5.08 \%$ transplanted cells, $n=5$ animals; Vgat $^{-1-}, 28.68 \pm 3.50 \%$ trans- 
A

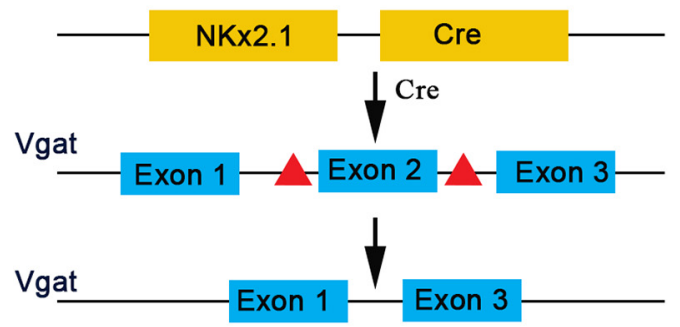

C
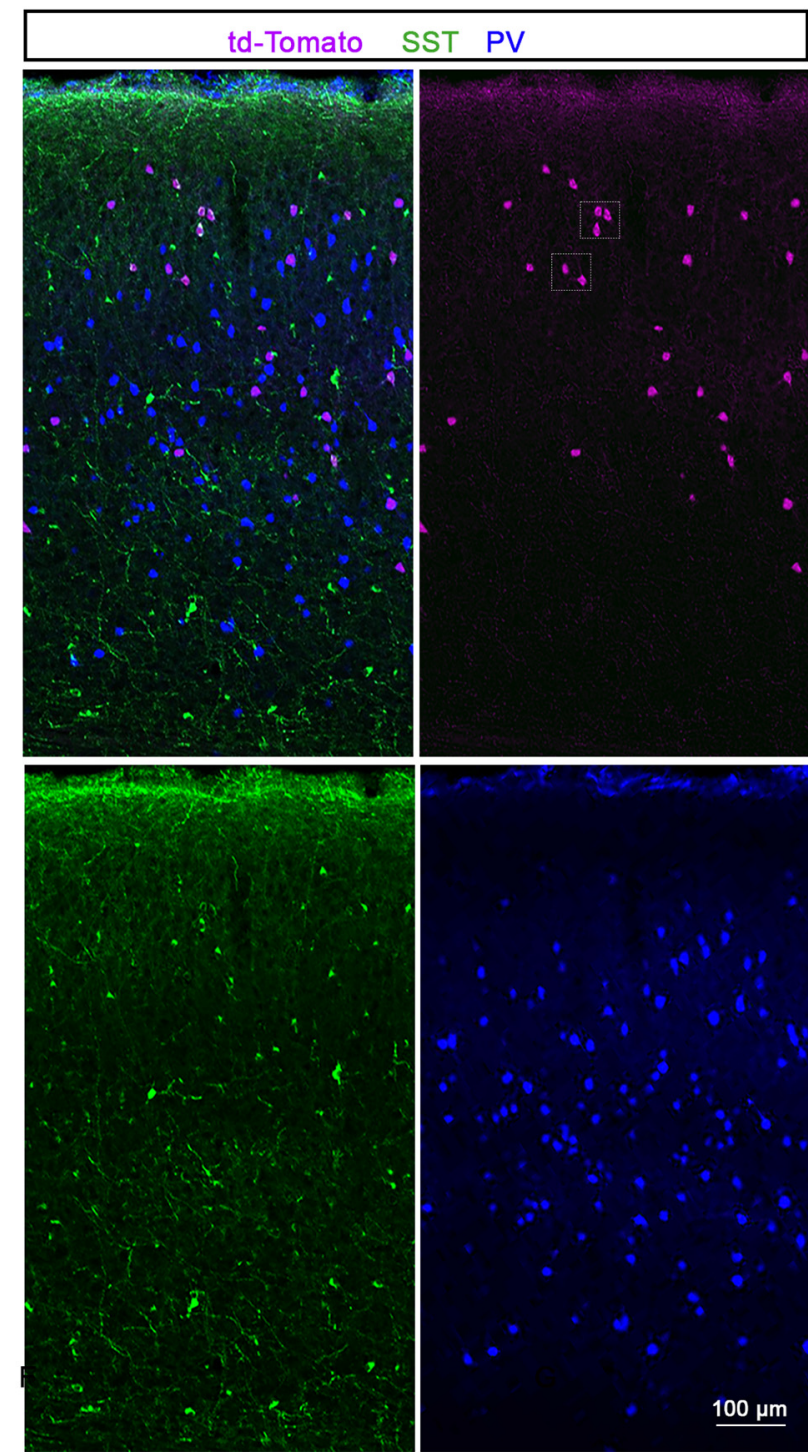

B

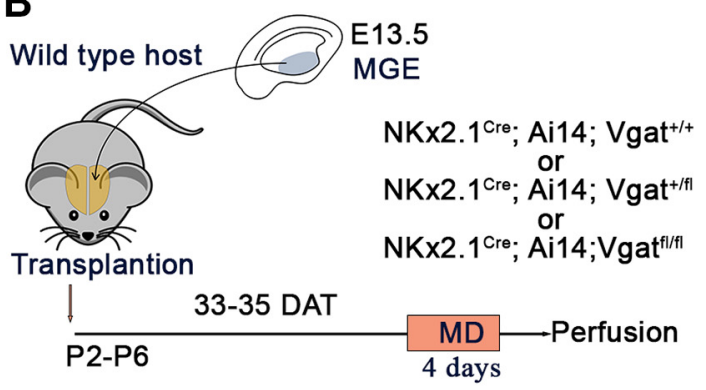

D
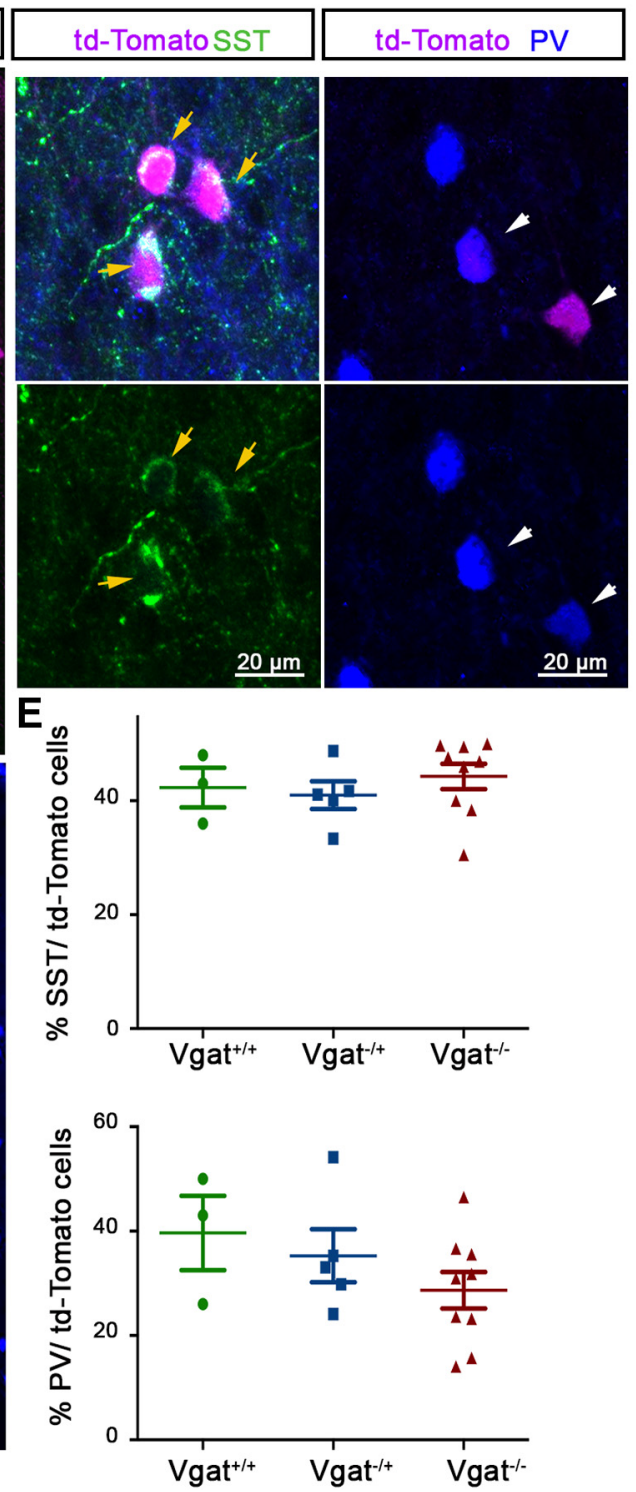

Figure 1. Transplanted MGE-derived CINs successfully differentiate and disperse in the host neocortex. $\boldsymbol{A}$, Experimental strategy showing that transgenic mice carrying V $\mathrm{gat}$ floxed allele were crossed with Nkx2.1-cre mouse driver line to conditionally knock out Vgat from MGE-derived interneurons. B, Cells were dissected from the MGE at E13.5, dissociated, and transplanted into the visual cortex of P2-P6 hosts. MGE transplantation recipient brains were examined for ODP at 33-35 DAT. C, Coronal sections were stained for Td-tomato, SST, and PV, which shows dispersion in the host visual cortex. D, Representative magnified image of the visual cortex showing colocalization between td-Tomato and SST in Vgat ${ }^{-1-}$ MGE cells (left) and td-Tomato and PV in Vgat ${ }^{-1-}$ MGE cells (right). $\boldsymbol{E}$, Percentage of transplanted SST (top) and PV (bottom) interneurons that express tdTomato in anatomically isolated MGE transplants.

planted cells, $n=9$ animals, one-way ANOVA, $p=0.2951)$. Their laminar distributions were similar among the three genotypes (Fig. 3B, Table 1). Transplanted $\mathrm{Vgat}^{+/+}, \mathrm{Vgat}^{+/-}$, and Vgat $^{-1-}$ MGE cells were found to disperse away from the injection site (Fig. 3A) to a similar extent, both along the rostrocaudal
(Fig. 3C, Table 2) and the mediolateral axes (Fig. 3D) $\left(\right.$ Vgat $^{+/+}$, $5.962 \pm 0.1161 \mathrm{~mm}, n=3 ;$ Vgat $^{+/-}, 4.133 \pm 0.7740 \mathrm{~mm}, n=3$; Vgat $^{-1-}, 4.936 \pm 0.2346 \mathrm{~mm}, n=3, p=0.0869$, one-way ANOVA). In addition, no significant differences were found in transplanted cell density across the 3 genotypes, demonstrating 


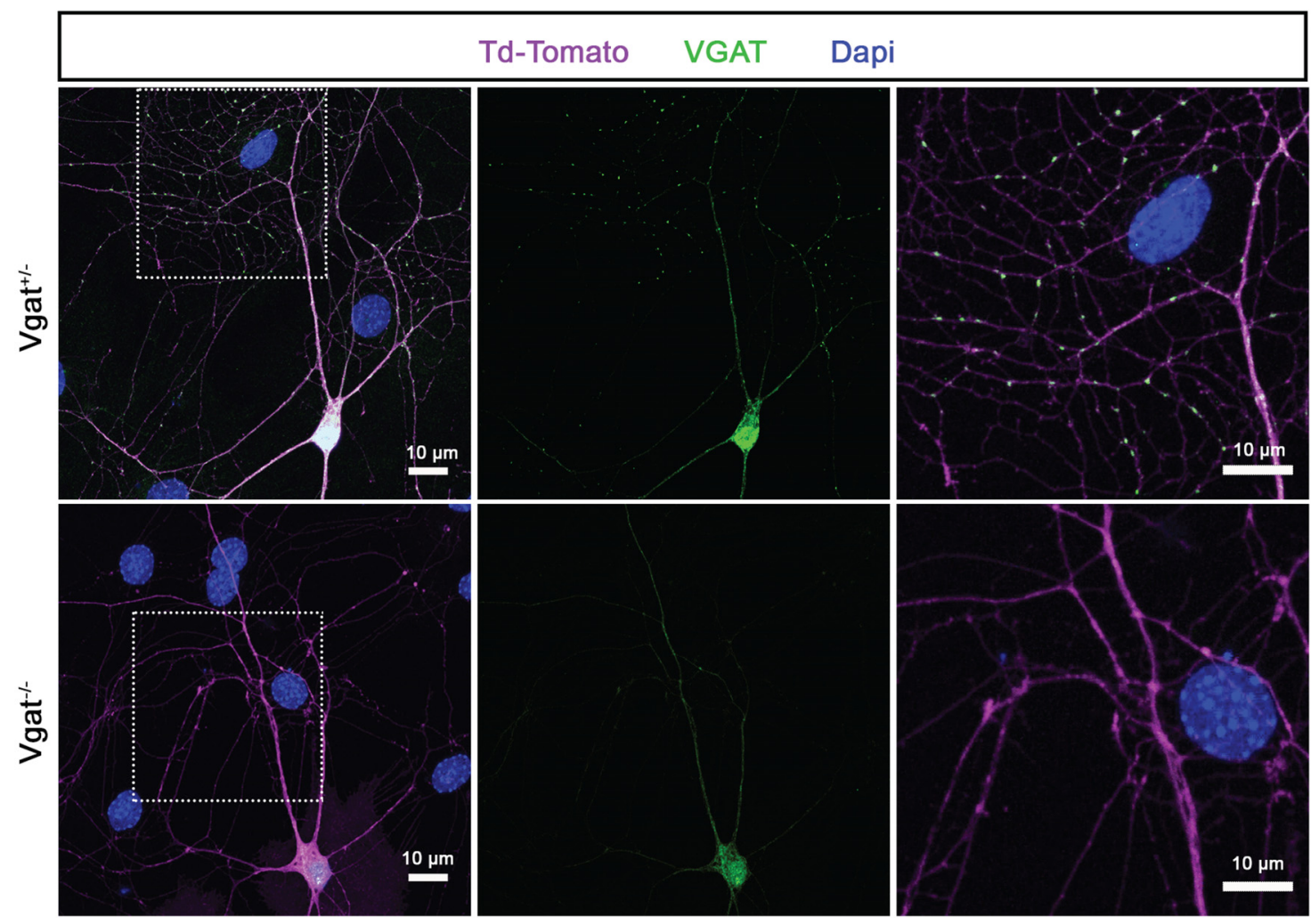

Figure 2. Representative image showing proof of concept of the experimental strategy. VGAT loss of function is confirmed by the lack of immunohistochemically demonstrable VGAT puncta from the MGE-derived cortical interneurons with Vgat ${ }^{-1-}$ allele compared with the $\mathrm{Vgat}^{+\prime-}$ allele. Interneurons in vitro were reacted for td-Tomato (magenta), VGAT (green), and stained with DAPI (blue). The VGAT antibody is a polyclonal antibody that stains the soma and the neurites to a limited extent even in the $\mathrm{Vgat}^{-1-}$ cells. The figure shows false colors in which antibody staining is rendered.

A

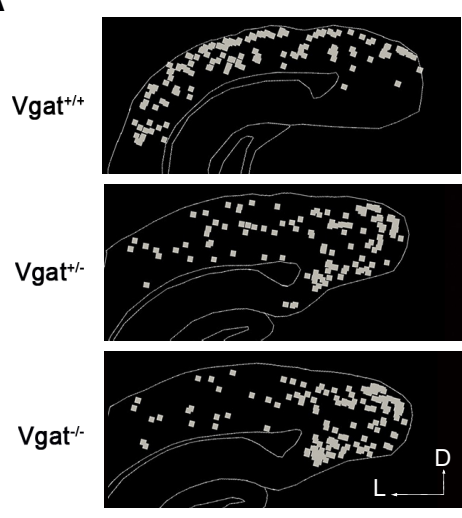

C

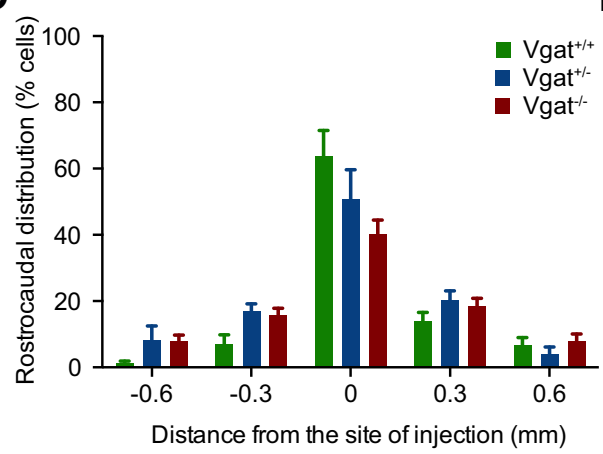

B

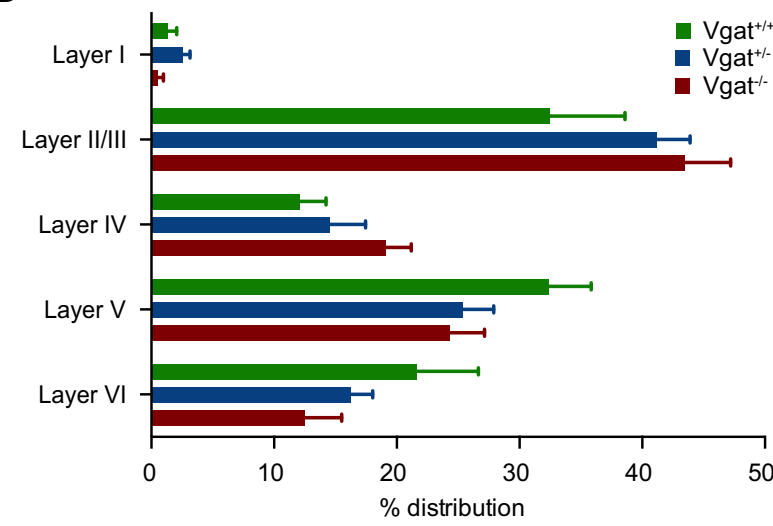

D

E
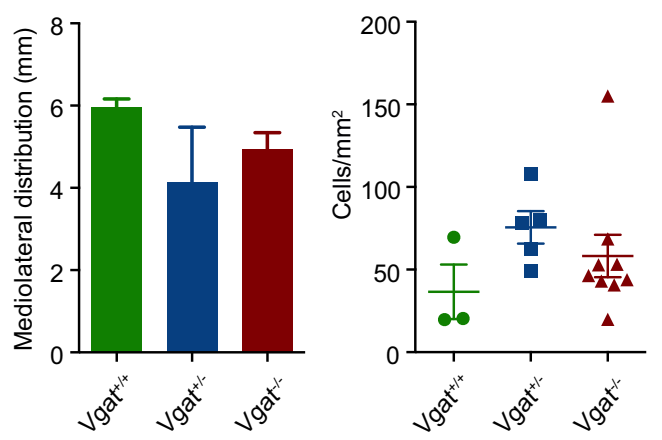

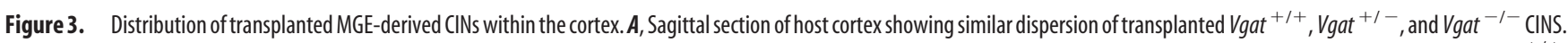
Distribution between the different layers of the cortex $(\boldsymbol{B})$ and the distribution from the injection site along the rostrocaudal axis $(\boldsymbol{C})$ and mediolateral axis $(\boldsymbol{D})$ were similar in transplanted $\mathrm{Vgat}{ }^{+/+}$, $\mathrm{Vgat}^{+1-}$, and $\mathrm{Vgat}^{-1-}$ CINS. E, Cell density was also comparable between the three genotypes. 
Table 1. One-way ANOVA analysis of laminar distributions from Figure 3B shown as mean \pm SEM cell percentages

\begin{tabular}{|c|c|c|c|c|}
\hline & Vgat $^{+1+}$ & Vgat $^{+1-}$ & Vgat $^{-1-}$ & $\begin{array}{l}\text { One-way ANOVA } \\
p \text {-value }\end{array}$ \\
\hline Layer I & $1.37 \pm 0.690(n=9)$ & $2.543 \pm 0.613(n=7)$ & $0.546 \pm 0.451(n=7)$ & 0.1155 \\
\hline Layer II/III & $32.45 \pm 6.138(n=9)$ & $41.23 \pm 2.661(n=7)$ & $43.50 \pm 3.695(n=7)$ & 0.2358 \\
\hline Layer IV & $12.10 \pm 2.135(n=9)$ & $14.59 \pm 2.860(n=7)$ & $19.14 \pm 2.049(n=7)$ & 0.1244 \\
\hline Layer V & $32.44 \pm 3.410(n=9)$ & $25.41 \pm 2.492(n=7)$ & $24.34 \pm 2.814(n=7)$ & 0.1344 \\
\hline Layer VI & $21.63 \pm 5.005(n=9)$ & $16.23 \pm 1.794(n=7)$ & $12.48 \pm 3.037(n=7)$ & 0.2558 \\
\hline
\end{tabular}

Table 2. One-way ANOVA analysis of rostrocaudal distribution from Figure 3 C shown as mean \pm SEM cell percentages

\begin{tabular}{|c|c|c|c|c|}
\hline $\begin{array}{l}\text { Distance from } \\
\text { injection site }\end{array}$ & Vgat $^{+1+}$ & Vgat $^{+1-}$ & Vgat $^{-1-}$ & $\begin{array}{l}\text { One-way ANOVA } \\
p \text {-value }\end{array}$ \\
\hline$-0.6 \mathrm{~mm}$ & $1.161 \pm 0.704(n=3)$ & $8.239 \pm 4.264(n=3)$ & $7.940 \pm 1.802(n=3)$ & 0.1928 \\
\hline$-0.3 \mathrm{~mm}$ & $6.974 \pm 2.813(n=3)$ & $16.97 \pm 2.211(n=3)$ & $15.78 \pm 2.083(n=3)$ & 0.05 \\
\hline $0 \mathrm{~mm}$ & $63.75 \pm 7.768(n=3)$ & $50.69 \pm 8.961(n=3)$ & $40.14 \pm 4.340(n=3)$ & 0.1512 \\
\hline $0.3 \mathrm{~mm}$ & $13.85 \pm 2.720(n=3)$ & $20.18 \pm 2.900(n=3)$ & $18.44 \pm 2.365(n=3)$ & 0.2970 \\
\hline $0.6 \mathrm{~mm}$ & $6.746 \pm 2.258(n=3)$ & $3.932 \pm 2.185(n=3)$ & $7.911 \pm 2.188(n=3)$ & 0.4708 \\
\hline
\end{tabular}

that Vgat deletion does not impair transplanted cell survival (Fig.

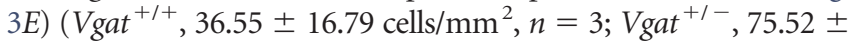
9.840 cells $/ \mathrm{mm}^{2}, n=5 ; \mathrm{Vgat}^{-1-}, 58.20 \pm 12.85 \mathrm{cells} / \mathrm{mm}^{2}, n=9$, one-way ANOVA, $p=0.3024$ ). Together, these results demonstrate that Vgat deletion does not impair the differentiation and spatial distribution of the heterochronically transplanted MGE cells.

We also assessed the presence of PNNs and Otx2 around and in PV-expressing MGE transplantation-derived cells. PNNs correspond to a specialized type of extracellular matrix highly enriched in chondroitin sulfate proteoglycans (CSPGs), which preferentially and progressively enwraps PV interneurons as they mature throughout the forebrain (Härtig et al., 1992, Köppe et al., 1997). Otx2 appearance in the visual cortex is activity dependent and its loss alters ODP onset (Sugiyama et al., 2008, Spatazza et al., 2013). Otx2 is specifically internalized by PV interneurons via its interaction with PNNs and is necessary for PV cell maturation (Beurdeley et al., 2012). The lectin WFA revealed PNNs in transplanted MGE cell recipient brain sections across all layers of the cortex. We found no difference in the percentage of transplanted $\mathrm{Vgat}^{+/+}, \mathrm{Vgat}^{+/-}$, and $\mathrm{Vgat}^{-1-} \mathrm{PV}$ cells that display PNNs as measured by WFA staining intensity (Fig. 4A, B) $\left(\right.$ Vgat $^{+/+} 76.67 \pm 14.53 \%, n=3$ mice; $V_{\text {gat }}{ }^{+/-}, 79.68 \pm 6.71 \%$, $n=4$ mice; Vgat $^{-1-}, 84.29 \pm 9.67 \%, n=3$ mice; ANOVA, $p=$ $0.8794)$. In addition, there was no difference in the PNN staining intensity, a product of area (in pixels) and mean gray value (Fig.

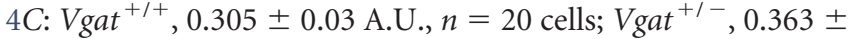
0.0322 A.U., $n=39$ cells; Vgat $^{-1-}, 0.375 \pm 0.04$ A.U., $n=40$ cells; ANOVA, $p=0.6255$ ) in animals perfused at $\mathrm{P} 46$ at the end of the second critical period. Similar levels of Otx2 protein were also found in the transplanted PV cells of the different genotypes (Fig. 4D, E: Vgat $^{+/+} 100.0 \pm 0.0 \%$ of PV cells contained Otx2, $n=7$ mice; Vgat ${ }^{+/-}, 100.0 \pm 0.0 \%$ of PV cells contained Otx2, $n=5$ mice; ggat $^{-1-}, 96.00 \pm 9.67 \%$ of PV cells contained Otx2, $n=5$ mice; ANOVA, $p=0.3206$ ) (Fig.

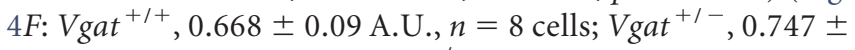
0.111 A.U., $n=10$ cells; Vgat ${ }^{-1-} 0.661 \pm 0.04$ A.U., $n=27$ cells; ANOVA, $p=0.6651)$. Together, these results show that transplanted MGE cells that are lacking Vgat can migrate into the heterochronic environment, differentiate into interneurons of their lineage, and acquire appropriate molecular characteristics including the expression of PNNs and incorporation of Otx2.
VGAT removal increases axonal and dendritic complexity

Results above indicate that synaptic GABA release from transplanted interneurons is not necessary for their dispersion or initial maturation. We next tested the impact of VGAT removal from transplanted interneurons on their morphology at 40-42 DAT. ggat $^{+/-}$or Vgat $^{-1-}$ E13.5 MGE cells were transplanted in the vicinity of the primary visual cortex (V1) of P6 WT hosts. Fifty-micrometer brain sections from recipient animals at $\mathrm{P} 46-$ P48 were immunostained for tdTomato and PV. PV-expressing, transplanted cells were observed in all layers of the visual cortex and their distribution and branching across these layers were similar between MGE-derived CINs from $\mathrm{Vgat}^{-1-}$ and $\mathrm{Vgat}^{+/-}$ transplants (Fig. 5). Axonal length and branch numbers of $\mathrm{Vgat}^{-1-}$ cells were, however, significantly greater than that of Vgat $^{+/+}$and Vgat $^{+/-}$cells (Fig. 6A,B; axonal length: Vgat $^{+/+}$, $750.1 \pm 109.4 \mu \mathrm{m}, n=14$ cells; Vgat $^{+\prime-}, 1645 \pm 358.4 \mu \mathrm{m}, n=$ 9 cells; Vgat $^{-1-}, 5345 \pm 978.5 \mu \mathrm{m}, n=10$ cells; significance was calculated using Bonferroni-corrected Mann-Whitney test. Comparison between $\mathrm{Vgat}^{+/+}$and $\mathrm{Vgat}^{+/-}, \mathrm{p}=0.0009$; comparison between $\mathrm{Vgat}^{+/+}$and $\mathrm{Vgat}^{-1-}, \mathrm{p}<0.0001$; comparison between $\mathrm{Vgat}^{+/-}$and $\mathrm{Vgat}^{-1-}, p=0.003$. Axonal nodes: Vgat $^{+/+}, 15.7 \pm 1.77 ;$ Vgat $^{+/-}, 40.8 \pm 5.27 ;$ Vgat $^{-1-}, 139.6 \pm$ 13.3; significance calculated using Bonferroni-corrected MannWhitney tests comparison between $\mathrm{Vgat}^{+/+}$and $\mathrm{Vgat}^{+/-}, \mathrm{p}<$ 0.0001; comparison between $\mathrm{Vgat}^{+/+}$and $\mathrm{Vgat}^{-1-}, p<0.0001$; comparison between $\mathrm{Vgat}^{+/-}$and $\left.\mathrm{Vgat}^{-1-}, \mathrm{p}<0.0001\right)$.

The mean length of dendritic segments between branch points was unchanged (Fig. 6D), but the integrated dendritic length of Vgat $^{-/-}$was greater than that of $\mathrm{Vgat}^{+/+} \mathrm{MGE}-$ derived CINs may be because of an increase in the number of branches (Fig. 6C,E; mean dendritic length: Vgat $^{+/+}, 208.4 \pm 26.14 \mu \mathrm{m}$; $\mathrm{Vgat}^{+/-}, 244.8 \pm 34.39 \mu \mathrm{m} ; \mathrm{Vgat}^{-/-}, 539.8 \pm 149.9 \mu \mathrm{m}$; significance calculated using Bonferroni-corrected Mann-Whitney tests, comparison between $\mathrm{Vgat}^{+/+}$and $\mathrm{Vgat}^{+/-}, \mathrm{p}=1.2012$; comparison between $\mathrm{Vgat}^{+/+}$and $\mathrm{Vgat}^{-1-}, p=0.780$; comparison between $\mathrm{Vgat}^{+1-}$ and $\mathrm{Vgat}^{-1-}, \mathrm{p}=0.28$; total dendritic length: $\mathrm{Vgat}^{+/+}, 1381 \pm 148.4 \mu \mathrm{m} ; \mathrm{Vgat}^{+/-}, 2039 \pm 399.3 \mu \mathrm{m}$; Vgat $^{-/-}, 2861 \pm 184.4 \mu \mathrm{m}$; significance calculated using Bonferroni-corrected Mann-Whitney tests, comparison between $\mathrm{Vgat}^{+/+}$and $\mathrm{Vgat}^{+/-}, \mathrm{p}=0.712$; comparison between $\mathrm{Vgat}^{+/+}$and $\mathrm{Vgat}^{-1-}, \mathrm{p}<0.0001$; comparison between $\mathrm{Vgat}^{+/-}$ and $V_{g a t}{ }^{-1-}, p=0.15$; dendritic nodes: $V_{g a t}^{+/+}, 27.9 \pm 3.076$; 
A

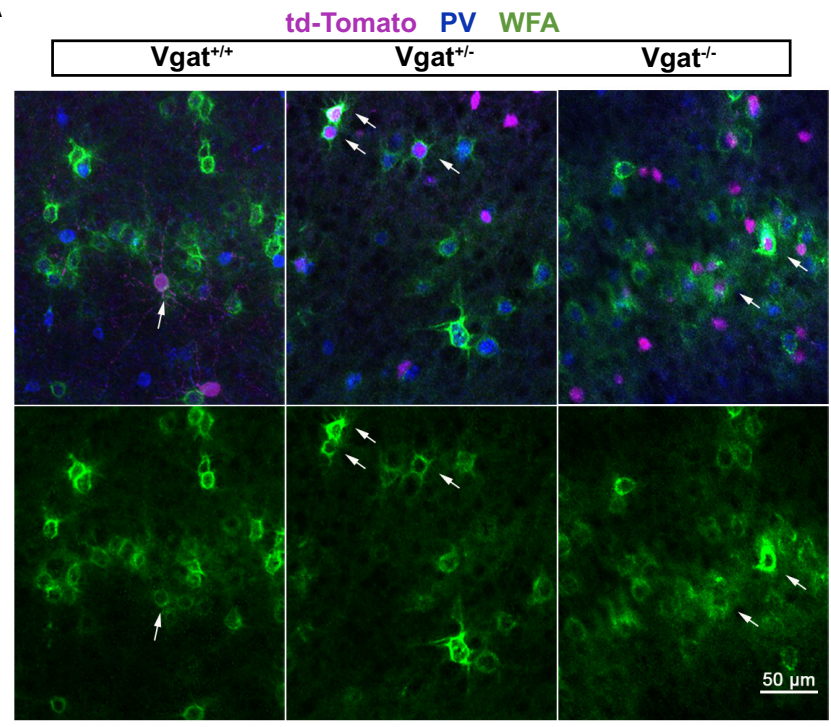

D

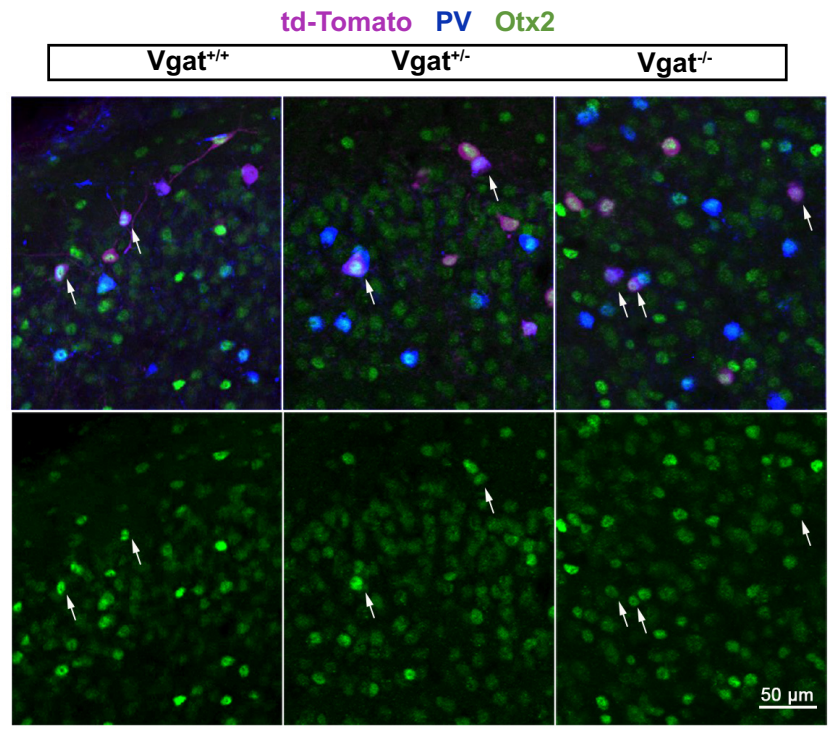

B

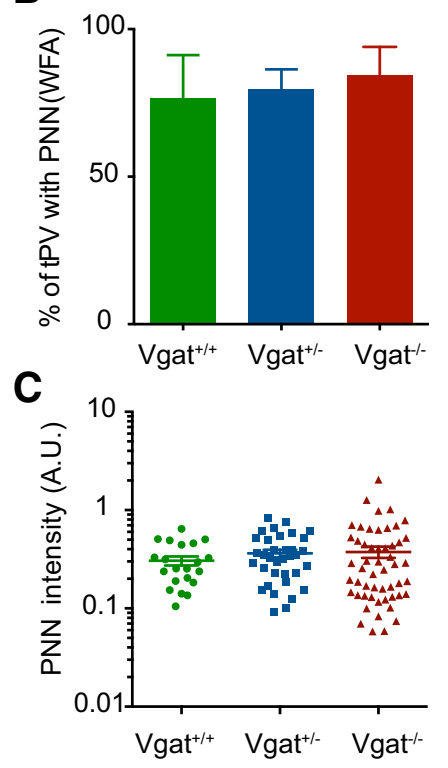

E

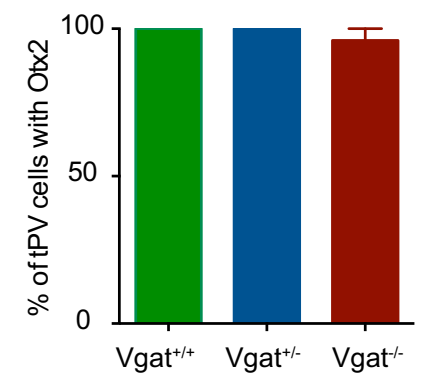

$\mathbf{F}$

Figure 4. Transplanted MGE-derived CINs show no change in the PNN density upon VGAT loss of function. $A$, Representative image of coronal sections in the binocular region of the visual cortex stained for td-Tomato (magenta), WFA (green), and PV (blue). B, C, Quantification shows no change in the percentage of transplanted PV cells (tPVs) expressing WFA or the pixel intensity (A.U.) of PNN stain in the tPVs between $\mathrm{ggat}^{+/+}, \mathrm{Vgat}^{+/-}$and $\mathrm{Vgat}{ }^{-/-}$alleles. $\boldsymbol{D}$, Intensity measure in transplanted interneurons is normalized to the average PNN levels in the host PV CINs in the same sections. Shown are coronal sections stained for td-Tomato (magenta), $0 \mathrm{tx2}$ (green), and PV (blue) in the visual cortex of the recipient animal. $\boldsymbol{E}, \boldsymbol{F}$, Quantification showing no significant change in the percentage of tPV expressing $0 \mathrm{tx} 2$ or the pixel intensity (A.U.) of Otx2 staining in the $\mathrm{PVs}$ in $\mathrm{Vgat}^{+/+}, \mathrm{Vgat}^{+/-}$, and $\mathrm{Vgat}^{-/-}$alleles. The intensity measure in transplanted interneurons is normalized to the average 0tx2 levels in the host PV CINs in the same sections.

Vgat $^{+/-}, 46.7 \pm 6.362 ;$ Vgat $^{-1-}, 105.9 \pm 12.74 ;$ significance calculated using Bonferroni-corrected Mann-Whitney tests, comparison between $\mathrm{Vgat}^{+/+}$and $\mathrm{Vgat}^{+/-}, p=0.0333$; comparison between $\mathrm{Vgat}^{+/+}$and $\mathrm{Vgat}^{-1-}, p<0.0001$; comparison between $\mathrm{Vgat}^{+/-}$and $\left.\mathrm{Vgat}^{-1-}, p=0.0048\right)$. Overall, the axons and dendrites of $\mathrm{Vgat}^{-1-}$ MGE transplanted interneurons were longer and more complex. This finding is consistent with previous studies and may be a consequence of decreased synaptic pruning in the absence of GABA neurotransmission (Wu et al., 2012).
VGAT removal increases capacitance, but had no effect on firing properties in vitro of transplanted MGE-derived CINs We investigated whether the VGAT knock-out altered physiological properties of MGE-derived CINs. Patch-clamp recordings from transplanted MGE-derived CINs labeled by tdTomato were performed at 38-40 DAT in acute cortical slices. No effects of VGAT expression on the normal development into non-fastspiking neurons (Fig. 7A) and fast-spiking neurons (Fig. 7B) (Butt et al., 2005) were observed. We categorized all recorded neurons as either fast spiking (spike rate $>90 \mathrm{~Hz}$ in response to 

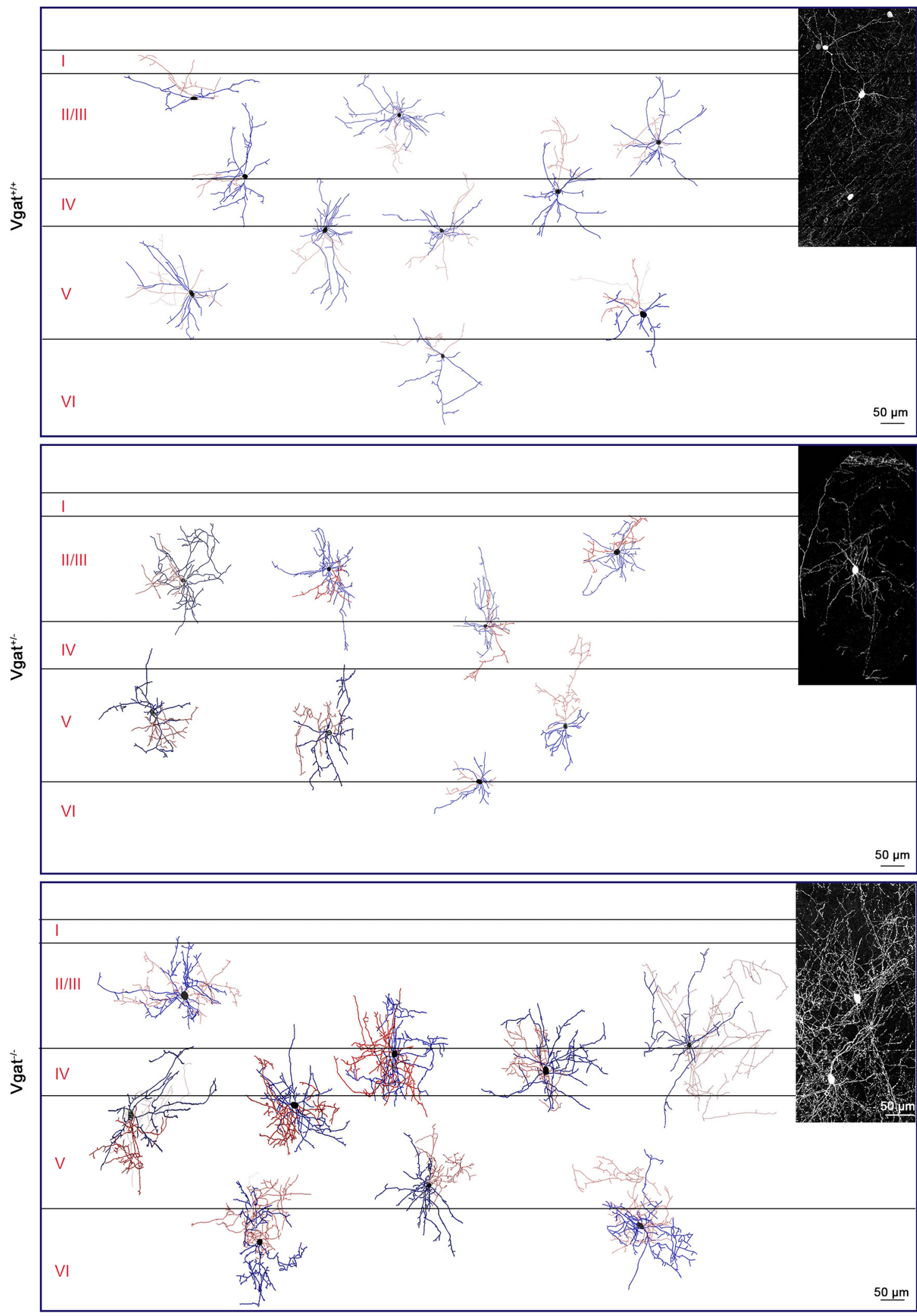

Figure 5. VGAT removal increases axonal and dendritic complexity of PV cells. Reconstruction of Vgat ${ }^{+/+}$(top), Vgat $^{+/-}$(middle), and Vgat ${ }^{-/-}$(bottom) PV-expressing interneurons in the host visual cortex shows greater morphological complexity after VGAT loss of function. Morphology of transplanted MGE-derived CINs is shown in the inset.

DC current injections of $2 \times$ rheobase) or non-fast spiking (Fig. $7 E$ ) (Larimer et al., 2017). Neither input resistance (Fig. 7F; non-FS mean \pm SEM: $363 \pm 33$ vs $468 \pm 202$ vs $232 \pm 94 \mathrm{M} \Omega$ for Vgat $^{+/+}$, Vgat $^{+/-}$, and Vgat $^{-/-}$respectively; FS: $176 \pm 15$ vs
$210 \pm 37$ vs $193 \pm 105 \mathrm{M} \Omega$ ) nor action potential threshold (Fig.

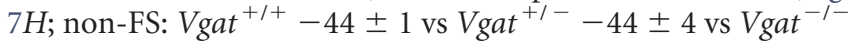

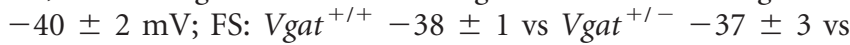
Vgat $^{-1-}-33 \pm 5 \mathrm{mV}$ ) nor rate of spontaneous EPSPs (Fig. 7J; 

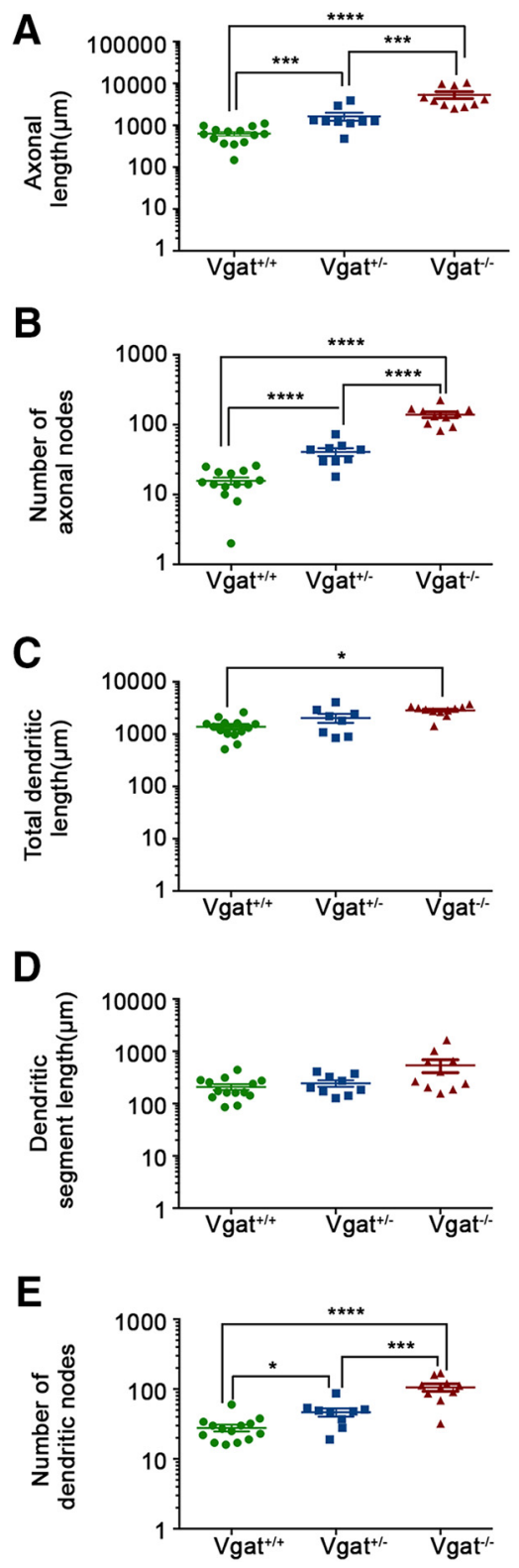

Figure 6. VGAT removal increases axonal and dendritic complexity. $A, B$, Measurements of axonal length $(\boldsymbol{A})$ and numbers of branches (nodes) $(\boldsymbol{B})$ showing increases in transplanted Vgat $^{-1-}$ MGE-derived CINs. C $\boldsymbol{E}$, Length of the dendritic segments is unchanged (D), but the complexity of the dendritic branches $(\boldsymbol{E})$ increases, resulting in an increase of integrated dendritic length $(C) .{ }^{* * *} p<0.0001,{ }^{* * *} p<0.001,{ }^{*} p<0.05$.

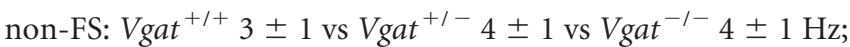
FS: Vgat $^{+1+} 10 \pm 1$ vs Vgat $^{+1-} 8 \pm 2$ vs ggat $^{-1-} 8 \pm 3 \mathrm{~Hz}$ ) was significantly different between genotypes. These properties that determine how likely a neuron is to fire action potentials were unaffected by VGAT deficiency. Two properties related to how often a neuron would be expected to release neurotransmitter, action potential width and max firing rate, were also unchanged in Vgat knock-out interneurons (action potential width: Fig. 7I;

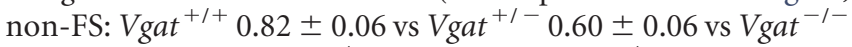

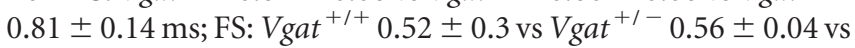
$\mathrm{Vgat}^{-1-} 0.48 \pm 0.05 \mathrm{~ms}$; maximum firing rate: non-FS: $\mathrm{Vgat}^{+/+}$

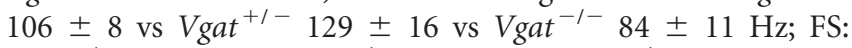

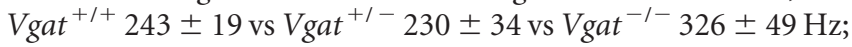
data not shown). The one physiological difference observed among the three genotypes of transplanted interneurons was

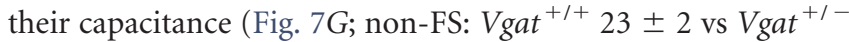

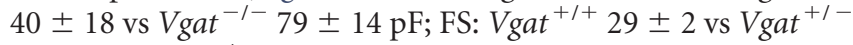

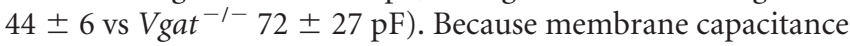
increases with increasing membrane area, the larger capacitances in neurons with fewer functional copies of VGAT are consistent with our findings of increased neurite length.

\section{MGE transplants lacking VGAT expression fail to induce a second critical period of ODP}

Previous work has shown that heterochronic transplantation of MGE-derived young interneurons into the neonatal or adult mouse visual cortex induces a second period of ODP (Southwell et al., 2010; Davis et al., 2015). We investigated whether VGAT removal from transplanted MGE cells affects the reactivation of plasticity in the host visual cortex. Recipients of $\mathrm{Vgat}^{+/+}$, $\mathrm{Vgat}^{+{ }^{-}}$, and $\mathrm{Vgat}^{-1-}$ E13.5 MGE cells were tested for ODP at 33-35 DAT using intrinsic signal optical imaging. Following 4-5 $\mathrm{d}$ of monocular deprivation, ggat $^{+/+}$and Vgat $^{+/-}$MGE cells induced robust plasticity as indicated by a shift in ODI. Magnitude of the ODI shift was similar between $\mathrm{Vgat}^{+/+}$and $\mathrm{Vgat}^{+1-}$ transplants (Fig. $8 A, B, D$; ODI shift, $V_{g a t}{ }^{+/+} 0.17 \pm 0.021$ A.U., $n=7$ mice vs ggat $^{+/-} 0.17 \pm 0.020$ A.U., $n=6$ mice; $p>0.05$, one-way ANOVA followed by multiple comparisons with Bonferroni correction). However, recipients of Vgat $^{-1-}$ MGE cells showed greatly decreased ODI shift compared with control groups (Fig. $8 C, D ; 0.04 \pm 0.006$ A.U., $n=10$ mice; $p<0.01$ vs Vgat $^{+1+}, p<0.001$ vs Vgat $^{+/-}$; one-way ANOVA followed by multiple comparisons with Bonferroni correction). Total transplantation-derived interneuron density was similar across the three recipient groups (Fig. $1 E$ ) and the baseline ODI between groups was also unaffected by transplantation (Fig. 9; gat $^{+/+}$, $0.252 \pm 0.0155$ A.U., $n=7$ mice; Vgat $^{+/-}, 0.245 \pm 0.0045$ A.U., $n=6$ mice; $V_{g a t}{ }^{-l-}, 0.233 \pm 0.0070$ A.U., $n=10$ mice; sham, $0.226 \pm 0.0065$ A.U., $n=5$ mice, one-way ANOVA, $p=0.3101$ ). Together, our results demonstrate that GABA release from transplanted MGE-derived CINs is essential for the induction of a second period of ODP despite their being functionally integrated into the host's neuronal circuit.

\section{Discussion}

This study addresses the role of VGAT and the associated vesicular release of GABA in the induction of a second, transplantation-mediated critical period. Transplanted interneurons derived from MGE progenitors that lack VGAT protein can migrate broadly, integrate into the host visual cortex, and retain their lineage-specific fate, as measured using anatomical location, marker expression, and electrophysiological properties. In addition, these neurons surrounded themselves with PNNs and incorporated Otx2, indicating that they are capable of maintaining stable synapses. However, despite successful integration into the host visual cortex, MGE transplants in which VGAT was knocked out failed to induce a second critical period following monocular deprivation. This demonstrates that the vesicular GABA transporter is essential for the transplanted neurons to induce plasticity.

Inhibitory interneurons migrate tangentially over long distances by detecting chemical cues in the extracellular environment. Previous studies have shown that tangentially migrating neurons in GAD67-GFP knock-in mice have a substantially slower migration rate, but no obvious disorders in gross neocortical organization have been observed after complete blockade of GABA synthesis in GAD65/GAD67 knock-out mice (Ji et al., 1999). GABA can promote migration via G-protein activation, mediated by $\mathrm{GABA}_{\mathrm{B}}$ receptors (Behar et al., 1998), and has a 


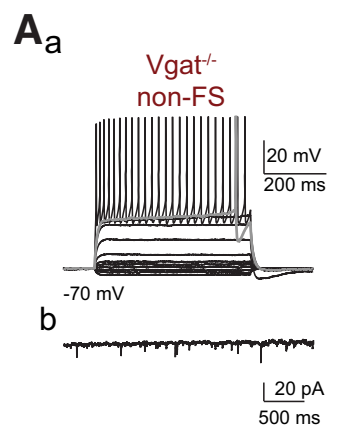

\section{E}

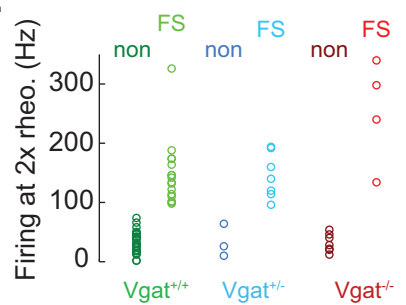

H

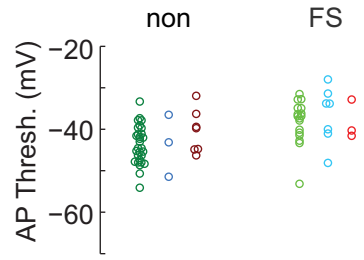

$B_{a}$

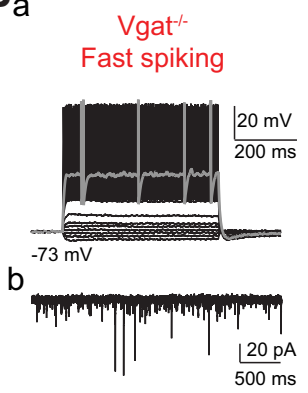

F

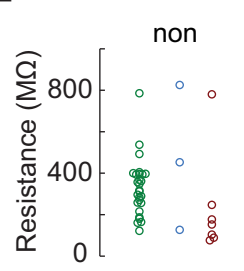

I

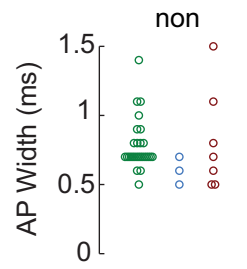

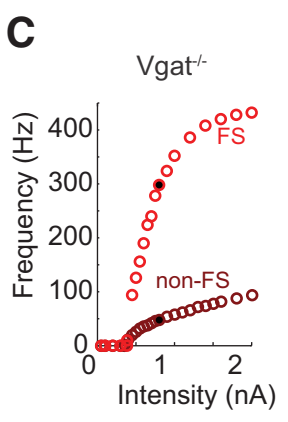

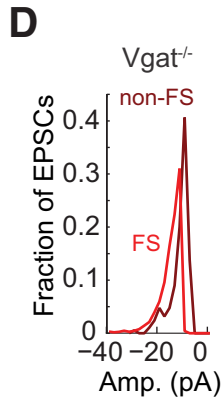

G
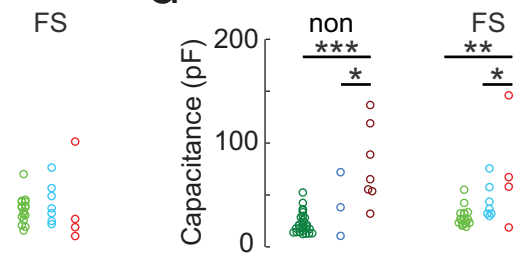

J

FS

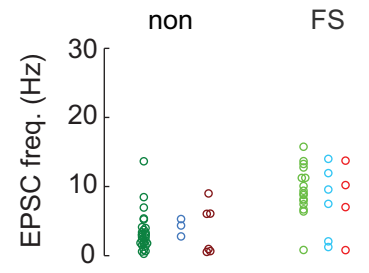

Figure 7. Transplantation-derived neurons lacking Vgat expression show typical electrophysiological properties of MGE-lineage interneurons. MGE transplants in which Vgat is constitutively not expressed (Gad2-Cre/+;Ai14/+;Vgat ${ }^{\mathrm{f} / f l}$ ) develop into typical non-fast spiking (non-FS) (A) and fast-spiking (FS) (B) interneurons as characterized by responses to intracellular current injections $(\boldsymbol{A} \boldsymbol{a}, \boldsymbol{B} \boldsymbol{a}$ : sample traces, bold trace is response at rheobase; $\boldsymbol{C}$ : - - I curves) and spontaneous EPSCs ( $\boldsymbol{A} \boldsymbol{b}, \boldsymbol{B} \boldsymbol{b}$ : sample traces recorded at $-70 \mathrm{mV}$; $\boldsymbol{D}$ : distribution of spontaneous EPSC amplitudes for example cells in $\boldsymbol{A}$ and $\boldsymbol{B})$. Compared with physiological properties of transplantation-derived interneurons with one (Gad2-Cre/+;Ai14/+;Vgat $\left.{ }^{+/ f l}\right)$ or two (Nkx2.1-Cre/+;Ai14/+;Vgat ${ }^{\mathrm{fl} / \mathrm{fl})}$ functional copies of $V$ gat, there were no significant differences in firing rate at $2 x$ rheobase $(\boldsymbol{E})$, input resistance $(\boldsymbol{F})$, action potential threshold $(\boldsymbol{H})$, action potential width $(\boldsymbol{I})$, or the frequency of spontaneous EPSCs $(\boldsymbol{J})$ (two-way ANOVA $p>0.05$ for all comparisons; are Vgat ${ }^{+/+}$, green; $V_{g a t}{ }^{+-}{ }^{\text {, blue; }}$. $g a t^{-/-}$, red). Membrane capacitance did differ between groups $\left(\mathbf{G} ; p<10^{-12}\right.$ for the genotype term of the two-way ANOVA) with significant differences seen between Vgat ${ }^{+/+}$and Vgat ${ }^{-I-}\left(p=0.0057\right.$ for non-FS, $p=0.016$ for FS). ${ }^{*} p<0.05,{ }^{* *} p<0.01{ }^{* * *} p<0.001$. Significance was calculated by false discovery-corrected two-way ANOVAs with $\alpha=0.05$ as a threshold for post hoc Mann-Whitney tests.

non-cell-autonomous effect on cortical interneurons. Consistent with these studies, we did not see significant changes in the final distribution of transplanted interneurons upon VGAT removal. The host cortex may provide sufficient GABA levels to maintain the migration of young interneurons. Similarly, we also did not see any effect of VGAT removal from the population of transplanted interneurons on the expression of SST or PV.

As young transplanted interneurons reach the different layers of cortex, they receive significant neuronal inputs and undergo synaptic maturation. The maturation of the cortex, and specifically PV interneurons, is associated with the deposition of CSPGs including PNNs, which are reduced by visual deprivation, indicating activity dependence (Lander et al., 1998; Pizzorusso et al., 2002; Carulli et al., 2010; Liu et al., 2013; Ye and Miao, 2013). The maturation of PNNs also requires the intracellular accumulation of Otx2 homeoprotein, which is internalized by PV interneurons via its interaction with PNNs (Sugiyama et al., 2008; Beurdeley et al., 2012). Intracellular levels of Otx2 are also thought to be experience dependent because they are detectable only after eye opening (Sugiyama et al., 2008).

Transplanted interneurons, like endogenous host interneurons, develop PNNs. Interestingly, the deposition of PNNs onto PV transplanted interneurons takes place at a faster rate compared with the endogenous timeline, occurring before the transplantation-mediated critical period (Bradshaw et al., 2018). The precocious timing of transplant PNN expression complicates the use of PNN as a measure of plasticity. However, given that both PNN deposition and Otx2 accumulation track the maturation of interneurons and are both activity dependent, they may be indicative of responsiveness to network activity. Interestingly, following VGAT removal, PNNs around graft-derived PV cells still formed. PNN density measured at P50, immediately after the closure of the transplantation-induced critical period, was similar to that surrounding host PV cells in all the groups studied: $\mathrm{Vgat}^{+/+}, \mathrm{Vgat}^{+/-}$, and $\mathrm{Vgat}^{-1-}$. Similar results were found for Otx2. Our results show that PNN formation and Otx2 accumulation in $\mathrm{Vgat}^{-1-}$ transplanted cortical interneurons occurs in the absence of induced plasticity. Because PNNs are heterogeneous (Matthews et al., 2002; Miyata et al., 2018), we cannot be certain that PNNs revealed by WFA staining that surround the transplanted $\mathrm{Vgat}^{-1-} \mathrm{PV}$ interneurons are identical to those of Vgat $^{+/+}$PV interneurons.

GABAergic signaling extends beyond the context of the synapse, affecting cell proliferation (LoTurco et al., 1995) and neurite growth (Spoerri, 1988). Reduction in GABA-mediated signaling by targeting either the enzymatic synthesis or vesicular release of GABA has an effect on interneuron innervation patterns. The reduction of GABA synthesis by targeting the expres- 
A

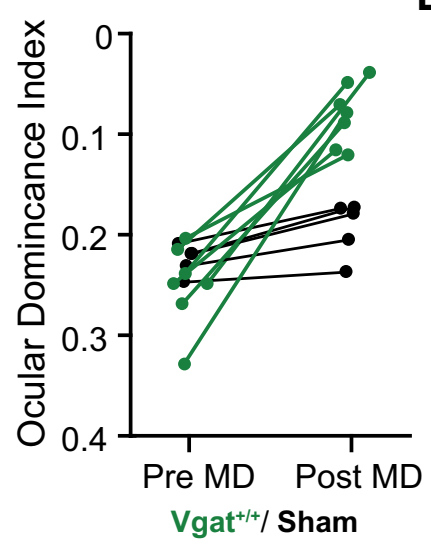

B

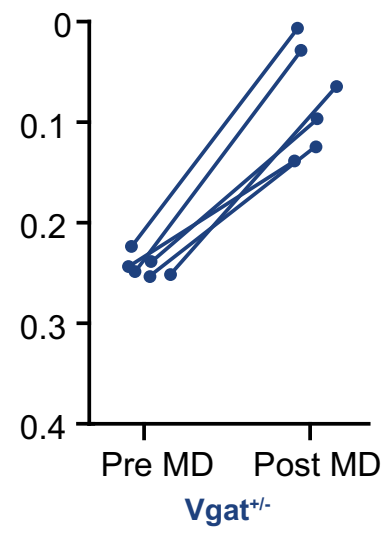

C

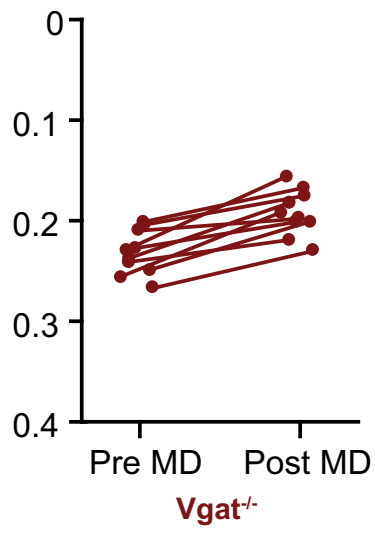

D

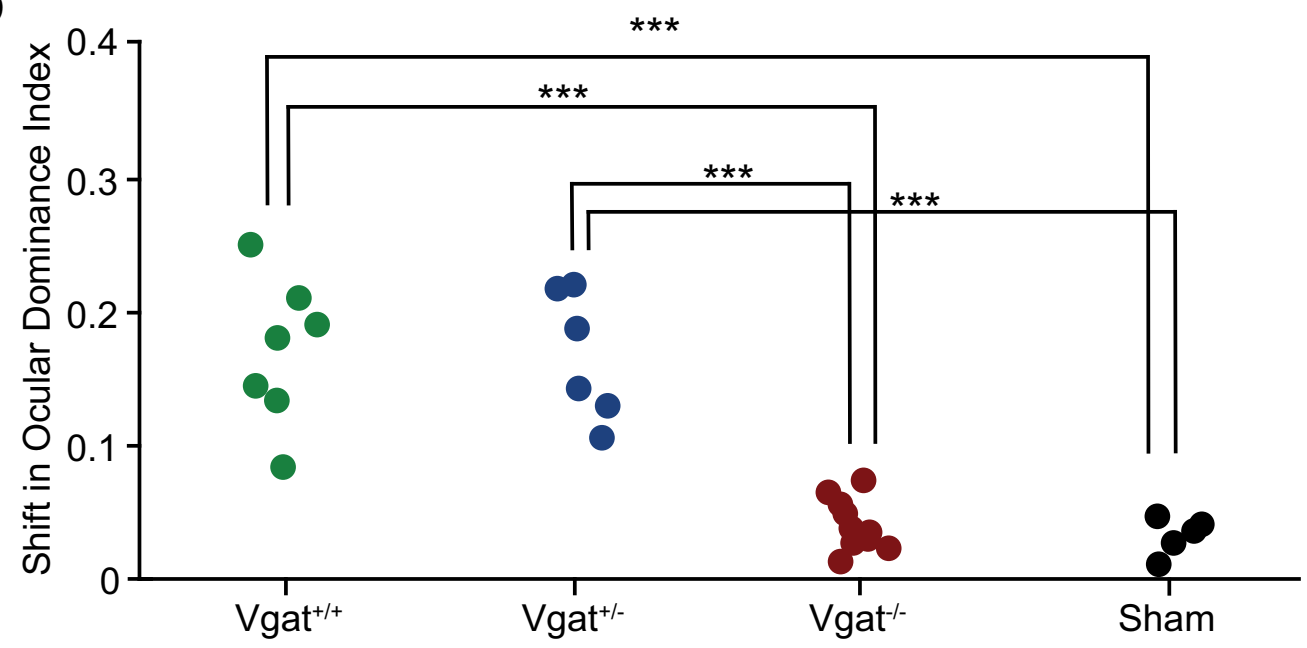

Figure 8. MGE transplants lacking Vgat expression fail to induce a second critical period of ODP. A-C, ODI (A.U.) measured before and after $4-5$ d of monocular deprivation of the contralateral eye. Significant plasticity was observed in $\mathrm{Vgat}^{+/+}\left(\boldsymbol{A}\right.$, green) and Vgat ${ }^{+/-}(\boldsymbol{B})$ MGE-cell-transplant recipients, but not in sham-transplant hosts (A, black). ODI shift (A.U.) in recipients of ( $\left.\boldsymbol{C}\right)$ $\mathrm{Vgat}^{-1-}$ neurons is greatly decreased. $\boldsymbol{D}$, Magnitudes of ODI change (A.U.) following deprivation. ${ }^{* *} p<0.001$. Significance was tested with one-way ANOVA followed by multiple comparisons with Bonferroni correction.

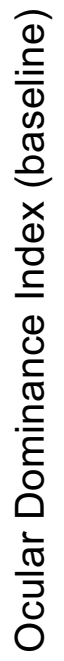

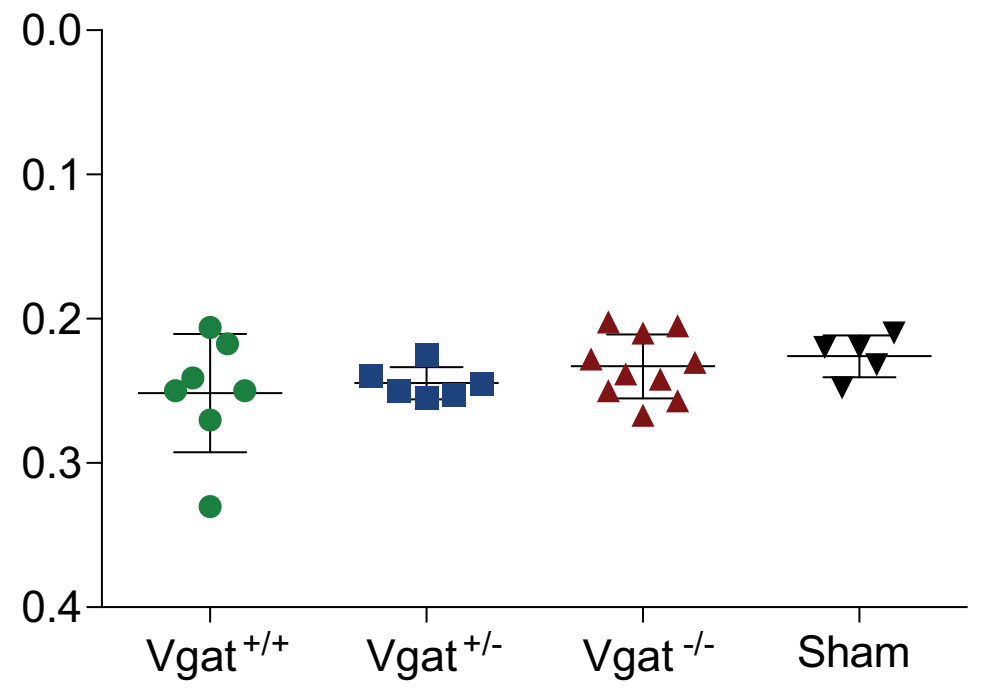

Figure 9. Visual cortex function was not affected by transplantation. There were no significant differences in the baseline ODI compared among $\mathrm{Vgat}^{+1+}, \mathrm{Vgat}^{+1-}, \mathrm{Vgat}^{-1-}$, and sham groups before monocular deprivation $\left(\mathrm{Vgat}^{+1+}, 0.252 \pm 0.016\right.$ $n=7 ;$ Vgat $^{+/-}, 0.245 \pm 0.005, n=6$; Vgat $^{-l-}, 0.233 \pm 0.007, n=10$; sham, $0.226 \pm 0.007$, one-way ANOVA, $p=$ $0.3101)$. sion of the synthetic enzyme GAD67 impedes the maturation of inhibitory synapses and axons (Chattopadhyaya et al., 2007), whereas the complete blockade of vesicle-mediated GABAergic signaling via the genetic ablation of vesicular GABA transporter (VGAT) or treatment of single cells with tetanus toxin light chain has been shown to increase axon and bouton density in those cells (Baho and Di Cristo, 2012; Wu et al., 2012). These results suggest that GABA-mediated synapse formation depends on GABA transmission for the elimination of new, tentative synaptic contacts. Our results in vivo are consistent with this observation. Upon VGAT removal in vivo, transplanted PV interneurons had longer and more complex axons and dendrites with more branch points. Consistent with this increase in membrane area, interneurons lacking VGAT function displayed increased membrane capacitance.

The ability of heterochronic transplants of PV or SST interneurons to 


\begin{tabular}{|c|c|c|c|}
\hline Genotype & $\begin{array}{l}\text { No. of } \\
\text { transplanted } \\
\text { interneurons }\end{array}$ & $\begin{array}{l}\text { Total no. of } \\
\text { endogenous } \\
\text { interneurons }\end{array}$ & $\begin{array}{l}\text { Fraction of transplanted } \\
\text { neurons of total } \\
\text { interneurons }\end{array}$ \\
\hline Vgat $^{-1-}$ & 219 & 991 & $22 \%$ \\
\hline Vgat $^{+1-}$ & 113 & 743 & $15.2 \%$ \\
\hline Vgat $^{+/-}$ & 73 & 1276 & $5.7 \%$ \\
\hline Vgat $^{-1-}$ & 90 & 602 & $14.9 \%$ \\
\hline Vgat $^{-1-}$ & 80 & 577 & $13.8 \%$ \\
\hline Vgat $^{-1-}$ & 144 & 1057 & $13.62 \%$ \\
\hline
\end{tabular}

induce a second critical period of juvenile-style plasticity precisely at the time in their maturation when endogenous inhibitory interneurons induce the native critical period suggests that transplantation-mediated plasticity involves an intrinsic, interneuron-specific mechanism. Such a mechanism is consistent with changes in the activity of GABAergic interneurons over the critical period (Kuhlman et al., 2013; Hengen et al., 2013; Figueroa Velez et al., 2017), which implicates the GABAergic interneurons causally in ODP. The GABAergic contribution made by the transplanted interneurons over the second critical period may play a similar role. However, the total amount of synaptic inhibition that the transplanted cells could provide, given that they may constitute only $5-22 \%$ (Table 3 ) of the inhibitory population in the host, may seem too small to have a major effect on the activity of the host circuit, especially given that much larger pharmacological perturbations of inhibition did not induce plasticity. Cortical diazepam infusion, for example, is known to double $\mathrm{GABA}_{\mathrm{A}}$ inhibitory currents, but does not trigger a second critical period (Hensch et al., 1998; Sigel and Steinmann, 2012). We therefore hypothesized that the transplanted cells might release a putative plasticizer substance other than GABA during the period of their maturation when a second period of plasticity is induced. The present finding that blocking vesicular GABA release from the transplanted interneurons abolishes transplantation-mediated plasticity provides no support for such a hypothesis. Because the genetic deletion of $\mathrm{Vgat}^{-I-}$ was under the control of Cre recombinase driven by the expression of the transcription factor $\mathrm{Nkx} 2.1$, we cannot rule out some GABAdependent process that is affected by the early loss of VGAT. However, we, and others, have shown that $\mathrm{Vgat}^{-1-}$ interneurons form normal synapses (Wu et al., 2012) and the functional state of the host visual cortex appeared intact in that baseline ODI measurements were similar among $\mathrm{Vgat}^{+/+}, \mathrm{Vgat}^{+/-}$, and $\mathrm{Vgat}^{-1-}$ transplants. Given that loss of VGAT results in the lack of plasticity at the time of the second critical period, these data suggest that the expression of VGAT over the second critical period, and most likely the inhibition that the transplanted cells produce, is necessary for transplantation-mediated ODP.

How, then, can we account for transplantation-induced plasticity, which we now know to depend on VGAT, and most likely the inhibition, that PV or SST cells produce? After all, the transplanted neurons provide only a small fraction of neurons in the circuit. The theory of attractor networks offers a possible explanation (Hopfield, 1982). The critical period may be thought of as an attractor state into which the developing cortical network settles and out of which perturbations such as monocular visual deprivation become no longer sufficient to move it, rather than a hard and fast period established by some biochemical mechanism. Even though the number of transplanted neurons is small, theoretical studies of recurrent network dynamics (Aljadeff et al., 2015) indicate that a small number of neurons can be have a disproportionately large impact on network dynamics. Such networks would then be significantly more responsive to external stimuli. Alternatively, the transplanted interneurons, despite their small numbers, might be sufficient to effectively inhibit the closed-eye pathways in V1 by themselves, thereby creating the second critical period. In this scenario, the strength of connection in the host circuitry would not need to change because all of the plasticity would be in the circuit created by the transplanted cells. In either case, studies of the activity of transplanted neurons around the second critical period of ODP promise to be informative.

Our results reveal that vesicular GABA release, as implied by the loss of the vesicular GABA transporter, is not necessary for the migration, specification, or integration of transplanted interneurons. Vesicular GABA release is also not required for the intracellular accumulation of Otx2 or the deposition of PNNs in the extracellular matrix. However, GABA transmission does play a role in limiting the arborization of transplanted interneurons and, as in the normal development of the visual cortex, GABA transmission from the transplanted interneurons is necessary for the induction of the second critical period.

\section{References}

Aljadeff J, Stern M, Sharpee T (2015) Transition to chaos in random networks with cell-type-specific connectivity. Phys Rev Lett 114:088101.

Alvarez-Dolado M, Calcagnotto ME, Karkar KM, Southwell DG, Jones-Davis DM, Estrada RC, Rubenstein JL, Alvarez-Buylla A, Baraban SC (2006) Cortical inhibition modified by embryonic neural precursors grafted into the postnatal brain. J Neurosci 26:7380-7389.

Anderson SA, Eisenstat DD, Shi L, Rubenstein JL (1997) Interneuron migration from basal forebrain to neocortex: dependence on dlx genes. Science 278:474-476.

Anderson SA, Marín O, Horn C, Jennings K, Rubenstein JL (2001) Distinct cortical migrations from the medial and lateral ganglionic eminences. Development 128:353-363.

Anderson S, Mione M, Yun K, Rubenstein JL (1999) Differential origins of neocortical projection and local circuit neurons: role of dlx genes in neocortical interneuronogenesis. Cereb Cortex 9:646-654.

Baho E, Di Cristo G (2012) Neural activity and neurotransmission regulate the maturation of the innervation field of cortical GABAergic interneurons in an age-dependent manner. J Neurosci 32:911-918.

Behar TN, Schaffner AE, Scott CA, O’Connell C, Barker JL (1998) Differential response of cortical plate and ventricular zone cells to GABA as a migration stimulus. J Neurosci 18:6378-6387.

Beurdeley M, Spatazza J, Lee HH, Sugiyama S, Bernard C, Di Nardo AA, Hensch TK, Prochiantz A (2012) Otx2 binding to perineuronal nets persistently regulates plasticity in the mature visual cortex. J Neurosci 32:9429-9437.

Bradshaw KP, Figueroa Velez DX, Habeeb M, Gandhi SP (2018) Precocious deposition of perineuronal nets on parvalbumin inhibitory neurons transplanted into adult visual cortex. Sci Rep 8:7480.

Brainard DH (1997) The Psychophysics Toolbox. Spat Vis 10:433-436.

Butt SJ, Fuccillo M, Nery S, Noctor S, Kriegstein A, Corbin JG, Fishell G (2005) The temporal and spatial origins of cortical interneurons predict their physiological subtype. Neuron 48:591-604.

Cang J, Kalatsky VA, Löwel S, Stryker MP (2005) Optical imaging of the intrinsic signal as a measure of cortical plasticity in the mouse. Vis Neurosci 22:685-691.

Carulli D, Pizzorusso T, Kwok JC, Putignano E, Poli A, Forostyak S, Andrews MR, Deepa SS, Glant TT, Fawcett JW (2010) Animals lacking link protein have attenuated perineuronal nets and persistent plasticity. Brain 133:2331-2347.

Chattopadhyaya B, Di Cristo G, Wu CZ, Knott G, Kuhlman S, Fu Y, Palmiter RD, ${ }^{* *}$ Huang ZJ (2007) GAD67-mediated GABA synthesis and signaling regulate inhibitory synaptic innervation in the visual cortex. Neuron 54:889-903. 
Davis MF, Figueroa Velez DX, Guevarra RP, Yang MC, Habeeb M, Carathedathu MC, Gandhi SP (2015) Inhibitory neuron transplantation into adult visual cortex creates a new critical period that rescues impaired vision. Neuron 86:1055-1066.

Di Cristo G, Chattopadhyaya B, Kuhlman SJ, Fu Y, Bélanger MC, Wu CZ, Rutishauser U, Maffei L, Huang ZJ (2007) Activity-dependent PSA expression regulates inhibitory maturation and onset of critical period plasticity. Nat Neurosci 10:1569-1577.

Ebihara S, Obata K, Yanagawa Y (2003) Mouse vesicular GABA transporter gene: genomic organization, transcriptional regulation and chromosomal localization. Brain Res Mol Brain Res 110:126-139.

Espinosa JS, Stryker MP (2012) Development and plasticity of the primary visual cortex. Neuron 75:230-249.

Fagiolini M, Hensch TK (2000) Inhibitory threshold for critical-period activation in primary visual cortex. Nature 404:183-186.

Fagiolini M, Fritschy JM, Löw K, Möhler H, Rudolph U, Hensch TK (2004) Specific GABAA circuits for visual cortical plasticity. Science 303:1681-1683.

Figueroa Velez DX, Ellefsen KL, Hathaway ER, Carathedathu MC, Gandhi SP (2017) Contribution of innate cortical mechanisms to the maturation of orientation selectivity in parvalbumin interneurons. J Neurosci 37:820-829.

Hanover JL, Huang ZJ, Tonegawa S, Stryker MP (1999) Brain-derived neurotrophic factor overexpression induces precocious critical period in mouse visual cortex. J Neurosci 19:RC40.

Härtig W, Brauer K, Brückner G (1992) Wisteria floribunda agglutininlabelled nets surround parvalbumin-containing neurons. Neuroreport 3:869-872.

Hengen KB, Lambo ME, Van Hooser SD, Katz DB, Turrigiano GG (2013) Firing rate homeostasis in visual cortex of freely behaving rodents. Neuron 80:335-342.

Hensch TK (2005) Critical period plasticity in local cortical circuits. Nat Rev Neurosci 6:877-888.

Hensch TK, Fagiolini M, Mataga N, Stryker MP, Baekkeskov S, Kash SF (1998) Local GABA circuit control of experience-dependent plasticity in developing visual cortex. Science 282:1504-1508.

Hirano AA, Liu X, Boulter J, Grove J, Pérez de Sevilla Müller L, Barnes S, Brecha NC (2016) Targeted deletion of vesicular GABA transporter from retinal horizontal cells eliminates feedback modulation of photoreceptor calcium channels. eNeuro 3:ENEURO.0148-15.2016.

Hopfield JJ (1982) Neural networks and physical systems with emergent collective computational abilities. Proc Natl Acad Sci U S A 79:25542558.

Isstas M, Teichert M, Bolz J, Lehmann K (2017) Embryonic interneurons from the medial, but not the caudal ganglionic eminence trigger ocular dominance plasticity in adult mice. Brain Struct Funct 222:539-547.

Ji F, Kanbara N, Obata K (1999) GABA and histogenesis in fetal and neonatal mouse brain lacking both the isoforms of glutamic acid decarboxylase. Neurosci Res 33:187-194.

Kalatsky VA, Stryker MP (2003) New paradigm for optical imaging: temporally encoded maps of intrinsic signal. Neuron 38:529-545.

Kaneko M, Stryker MP (2014) Sensory experience during locomotion promotes recovery of function in adult visual cortex. Elife 3:e 02798.

Köppe G, Brückner G, Brauer K, Härtig W, Bigl V (1997) Developmental patterns of proteoglycan-containing extracellular matrix in perineuronal nets and neuropil of the postnatal rat brain. Cell Tissue Res 288:33-41.

Kuhlman SJ, Olivas ND, Tring E, Ikrar T, Xu X, Trachtenberg JT (2013) A disinhibitory microcircuit initiates critical-period plasticity in the visual cortex. Nature 501:543-546.

Lander C, Zhang H, Hockfield S (1998) Neurons produce a neuronal cell surface-associated chondroitin sulfate proteoglycan. J Neurosci 18:174183.

Larimer P, Spatazza J, Espinosa JS, Tang Y, Kaneko M, Hasenstaub AR, Stryker MP, Alvarez-Buylla A (2016) Caudal ganglionic eminence precursor transplants disperse and integrate as lineage-specific interneurons but do not induce cortical plasticity. Cell Rep 16:1391-1404.

Larimer P, Spatazza J, Stryker MP, Alvarez-Buylla A, Hasenstaub AR (2017) Development and long-term integration of MGE-lineage cortical interneurons in the heterochronic environment. J Neurophysiol 118:131139.

Lin TW, Tan W, Barik A, Yin D-M, Brudvik E, Wang H, Xiong W-C, Mei L
(2018) Regulation of Synapse Development by Vgat Deletion from ErbB4-Positive Interneurons. J Neurosci 38:2533-2550.

Liu H, Xu H, Yu T, Yao J, Zhao C, Yin ZQ (2013) Expression of perineuronal nets, parvalbumin and protein tyrosine phosphatase $\sigma$ in the rat visual cortex during development and after BFD. Curr Eye Res 38:1083-1094.

LoTurco JJ, Owens DF, Heath MJ, Davis MB, Kriegstein AR (1995) GABA and glutamate depolarize cortical progenitor cells and inhibit DNA synthesis. Neuron 15:1287-1298.

Matthews RT, Kelly GM, Zerillo CA, Gray G, Tiemeyer M, Hockfield S (2002) Aggrecan glycoforms contribute to the molecular heterogeneity of perineuronal nets. J Neurosci 22: 7536-7547.

Miyata S, Nadanaka S, Igarashi M, Kitagawa H (2018) Structural variation of chondroitin sulfate chains contributes to the molecular heterogeneity of perineuronal nets. Front Integr Neurosci 12:3.

Nery S, Fishell G, Corbin JG (2002) The caudal ganglionic eminence is a source of distinct cortical and subcortical cell populations. Nat Neurosci 5:1279-1287.

Niell CM, Stryker MP (2008) Highly selective receptive fields in mouse visual cortex. J Neurosci 28:7520-7536.

Parnavelas JG (2000) The origin and migration of cortical neurones: new vistas. Trends Neurosci 23:126-131.

Pei Z, Chen Q, Koren D, Giammarinaro B, Acaron Ledesma H, Wei W (2015) Conditional knock-out of vesicular GABA transporter gene from starburst amacrine cells reveals the contributions of multiple synaptic mechanisms underlying direction selectivity in the retina. J Neurosci 35 13219-13232.

Pelli DG (1997) The VideoToolbox software for visual psychophysics: transforming numbers into movies. Spat Vis 10:437-442.

Pizzorusso T, Medini P, Berardi N, Chierzi S, Fawcett JW, Maffei L (2002) Reactivation of ocular dominance plasticity in the adult visual cortex. Science 298:1248-1251.

Pleasure SJ, Anderson S, Hevner R, Bagri A, Marin O, Lowenstein DH, Rubenstein JL (2000) Cell migration from the ganglionic eminences is required for the development of hippocampal GABAergic interneurons. Neuron 28:727-740.

Saito K, Kakizaki T, Hayashi R, Nishimaru H, Furukawa T, Nakazato Y, Takamori S, Ebihara S, Uematsu M, Mishina M, Miyazaki J, Yokoyama M, Konishi S, Inoue K, Fukuda A, Fukumoto M, Nakamura K, Obata K, Yanagawa Y (2010) The physiological roles of vesicular GABA transporter during embryonic development: a study using knock-out mice. Mol Brain 3:40.

Sigel E, Steinmann ME (2012) Structure, function, and modulation of GABAA receptors. J Biol Chem 287:40224-40231.

Southwell DG, Froemke RC, Alvarez-Buylla A, Stryker MP, Gandhi SP (2010) Cortical plasticity induced by inhibitory neuron transplantation. Science 327:1145-1148.

Southwell DG, Nicholas CR, Basbaum AI, Stryker MP, Kriegstein AR, Rubenstein JL, Alvarez-Buylla A (2014) Interneurons from embryonic development to cell-based therapy. Science 344:1240622.

Spatazza J, Lee HH, Di Nardo AA, Tibaldi L, Joliot A, Hensch TK, Prochiantz A (2013) Choroid-plexus-derived Otx2 homeoprotein constrains adult cortical plasticity. Cell Rep 3:1815-1823.

Spatazza J, Mancia Leon WR, Alvarez-Buylla A (2017) Transplantation of GABAergic interneurons for cell-based therapy. Prog Brain Res 231: $57-85$.

Spoerri PE (1988) Neurotrophic effects of GABA in cultures of embryonic chick brain and retina. Synapse 2:11-22.

Sugiyama S, Di Nardo AA, Aizawa S, Matsuo I, Volovitch M, Prochiantz A, Hensch TK (2008) Experience-dependent transfer of Otx2 homeoprotein into the visual cortex activates postnatal plasticity. Cell 134:508-520.

Tang Y, Stryker MP, Alvarez-Buylla A, Espinosa JS (2014) Cortical plasticity induced by transplantation of embryonic somatostatin or parvalbumin interneurons. Proc Natl Acad Sci U S A 111:18339-18344.

Tong Q, Ye CP, Jones JE, Elmquist JK, Lowell BB (2008) Synaptic release of GABA by AgRP neurons is required for normal regulation of energy balance. Nat Neurosci 11:998-1000.

Tyson JA, Anderson SA (2014) GABAergic interneuron transplants to study development and treat disease. Trends Neurosci 37:169-177.

Vong L, Ye C, Yang Z, Choi B, Chua S Jr, Lowell BB (2011) Leptin action on GABAergic neurons prevents obesity and reduces inhibitory tone to POMC neurons. Neuron 71:142-154.

Weaver DR, van der Vinne V, Giannaris EL, Vajtay TJ, Holloway KL, Anaclet 
C (2018) Functionally complete excision of conditional alleles in the mouse suprachiasmatic nucleus by Vgat-Ires-Cre. J Biol Rhythms 33:179-191.

Wehr M,Zador AM (2003) Balanced inhibition underlies tuning and sharpens spike timing in auditory cortex. Nature 426:442-446.

Wichterle H, Garcia-Verdugo JM, Herrera DG, Alvarez-Buylla A (1999) Young neurons from medial ganglionic eminence disperse in adult and embryonic brain. Nat Neurosci 2:461-466.

Wichterle H, Turnbull DH, Nery S, Fishell G, Alvarez-Buylla A (2001) In utero fate mapping reveals distinct migratory pathways and fates of neurons born in the mammalian basal forebrain. Development 128:3759-3771.

Wojcik SM, Katsurabayashi S, Guillemin I, Friauf E, Rosenmund C, Brose N, Rhee JS (2006) A shared vesicular carrier allows synaptic corelease of GABA and glycine. Neuron 50:575-587.
Wonders CP, Anderson SA (2006) The origin and specification of cortical interneurons. Nat Rev Neurosci 7:687-696.

Wu X, Fu Y, Knott G, Lu J, Di Cristo G, Huang ZJ (2012) GABA signaling promotes synapse elimination and axon pruning in developing cortical inhibitory interneurons. J Neurosci 32:331-343.

Xu Q, Tam M, Anderson SA (2008) Fate mapping Nkx2.1-lineage cells in the mouse telencephalon. J Comp Neurol 506:16-29.

Ye Q, Miao QL (2013) Experience-dependent development of perineuronal nets and chondroitin sulfate proteoglycan receptors in mouse visual cortex. Matrix Biol 32:352-363.

Yizhar O, Fenno LE, Prigge M, Schneider F, Davidson TJ, O'Shea DJ, Sohal VS, Goshen I, Finkelstein J, Paz JT, Stehfest K, Fudim R, Ramakrishnan C, Huguenard JR, Hegemann P, Deisseroth K (2011) Neocortical excitation/inhibition balance in information processing and social dysfunction. Nature 477:171-178. 